\title{
Chemie finde ich eigentlich interessant, aber manchmal auch nicht - Zusammenhänge zwischen Merkmalen des Chemieunterrichts und dem situationalen Interesse von Lernenden
}

\author{
Sabrina Ochsen (D) - Sascha Bernholt $(\mathbb{D}) \cdot$ Andrea Bernholt $(\mathbb{D}) \cdot$ \\ Ilka Parchmann
}

Eingegangen: 11. November 2019 / Überarbeitet: 2. August 2021 / Angenommen: 1. Oktober 2021 / Online publiziert: 28. Oktober 2021

(C) Der/die Autor(en) 2022

Zusammenfassung Interesse ist ein wichtiges unterrichtliches Ziel und positiv mit weiteren Konstrukten wie Leistung und Selbstkonzept assoziiert. Eine Abnahme des Interesses von Lernenden in den Naturwissenschaften allgemein und insbesondere im Chemieunterricht über den Verlauf der Sekundarstufe wurde wiederholt festgestellt. Um Interesse im Schulkontext zu fördern, bietet sich gemäß des Vier-Phasen Modells der Interessenentwicklung insbesondere das zeitlich instabile situationale Interesse an. Die Befundlage zu möglichen Triggern für situationales Interesse ist sehr vielfältig, jedoch teilweise uneindeutig. Zudem werden bislang in den Untersuchungen zum situationalen Interesse zentrale Qualitäts- und Gestaltungsmerkmale von Unterricht wie die Klassenführung oder Unterrichtsphasierung nicht systematisch berücksichtigt. Dieses Desiderat adressiert die vorliegende Beobachtungsstudie im Fach Chemie, indem sie den Zusammenhang zwischen Unterrichtsqualität sowie -gestaltung mit dem situationalen Interesse von Lernenden der Sekundarstufe I mittels einer Mehrebenenanalyse untersucht. Die Ergebnisse bestätigen empirisch, dass in der vorliegenden Studie praktische Erarbeitungsphasen in positiver Relation zum situationalen Interesse der Lernenden stehen, während theoretische Erarbeitungsphasen damit negativ verknüpft sind. Einstiegs- und Sicherungsphasen weisen keinen systematischen Zusammenhang mit dem situationalen Interesse auf. Im Bereich der Unterrichtsqualität zeigen sich negative Zusammenhänge zwischen dem situationalen Interesse und Klassenmanagement bzw. Verständnisorientierung, während zwischen dem situationalen Interesse und Strukturierung bzw. Förderung positive Zusammenhänge festgestellt wurden. Mögliche Ursachen sowie Implikationen für Forschung und Praxis werden diskutiert.

Sabrina Ochsen $(\triangle) \cdot$ Sascha Bernholt $\cdot$ Andrea Bernholt $\cdot$ Ilka Parchmann Leibniz-Institut für die Pädagogik der Naturwissenschaften und Mathematik, Olshausenstr. 62, 24118 Kiel, Deutschland

E-Mail: ochsen@leibniz-ipn.de 
Schlüsselwörter Situationales Interesse $\cdot$ Beobachtungsstudie ·

Unterrichtsqualität · Mehrebenenanalyse · Unterrichtsphasen

\title{
I actually find chemistry interesting, but sometimes not-couplings between characteristics of chemistry lessons and students' situational interest
}

\begin{abstract}
Interest is an important learning goal and positively related to other variables like achievement and future vocational choices. However, a decrease of students' interest over the course of secondary school in STEM-related subjects is reported repeatedly. According to the Four-Phase Model of Interest Development, situational interest, which is a psychological state of attention, is an adequate focus to promote student interest. Research in the field of possible triggers is extensive and various. In this context, little is known about relations between instructional quality and specific sequences of lessons like practical explore-phases, respectively, and students' situational interest. In order to gain knowledge about these interrelations, an observational study in chemistry lessons was conducted. Based on classroom observations, a multilevel analysis was used to examine potential couplings. Regarding instructional quality, negative relations were detected for classroom management and understanding orientation, while positive relations were found for structuring and fostering. Moreover, experimental phases were shown to have a positive coupling to students' situational interest, while theoretical phases seem to have a negative one. No systematic relations were noted for engage and explain phases. Possible implications for research and teaching in schools are discussed.
\end{abstract}

Keywords Situational interest · Observational study · Instructional quality · Multilevel analysis · Instructional phases

\section{Einleitung}

Die zentrale Bedeutung von Interesse im (naturwissenschaftlichen) Unterricht ist in der Bildungsforschung allgemein bekannt und akzeptiert. Interesse wird positiv mit Leistung, Selbstkonzept, Motivation und Selbstwirksamkeitserwartungen assoziiert (Potvin und Hasni 2014). Neben Faktoren wie der Leistung und dem Selbstkonzept der Lernenden wird auch das Interesse positiv mit den Berufs- und Kurswahlentscheidungen assoziiert (Eitemüller und Walpuski 2018; Maltese und Tai 2011). So fasste Su (2018) in ihrem Review zusammen, dass das Interesse prädiktiv für die Berufswahl ist und positiv mit den aufgebrachten Anstrengungen im Beruf korreliert.

Obwohl Lernende häufig mit einem hohen Interesse an Naturwissenschaften in die Sekundarstufe kommen, hält dieses Interesse nicht an (Potvin und Hasni 2014). Eine differenzierte Betrachtung von Interesse bestärkt diesen Befund: So lässt sich im Chemieunterricht in verschiedenen naturwissenschaftsspezifischen Aktivitäten eine Abnahme des Interesses beobachten (Höft et al. 2019). Ein möglicher Erklärungsansatz für diesen Rückgang des Interesses an den Naturwissenschaften ist die Interessendifferenzierung, welche im Zuge der Pubertät stattfindet. Allerdings sinkt 
das Interesse in den Naturwissenschaften Chemie und Physik im Vergleich mit anderen Unterrichtsfächern überdurchschnittlich stark (Krapp und Prenzel 2011). Schülerinnen sind von diesem Abfall stärker betroffen als Schüler. Diese Befunde bieten einen Erklärungsansatz für die verhältnismäßig geringe Zahl an Lernenden, die sich nach ihrem Schulabschluss für einen naturwissenschaftlichen Beruf entscheiden (Lyons und Quinn 2010). Gerade unter Einbezug der steigenden Berufsmöglichkeiten im Bereich Mathematik, Informatik, Naturwissenschaften und Technik (MINT) gewinnt dieser Aspekt an Bedeutung. Hieraus ergibt sich eine Herausforderung für die Bildungsforschung: Es muss nach Ansätzen und Möglichkeiten gesucht werden, das Interesse von Lernenden explizit zu fördern bzw. bei bereits naturwissenschaftlich interessierten Lernenden aufrecht zu halten. Für eine effiziente Weiterentwicklung von Chemieunterricht wird in dieser Studie zunächst explorativ untersucht, welche Ansätze zur Interessenförderung bereits realisiert worden sind und wie sie auf das Interesse der Lernenden wirken.

\section{Theoretischer Hintergrund und Stand der Forschung}

Interesse lässt sich mittels unterschiedlicher theoretischer Ansätze definieren. Gemeinsam haben die meisten Definitionen, dass es sich bei Interesse um ein multidimensionales Konstrukt handelt, welches sowohl affektive als auch kognitive Bestandteile beinhaltet (Krapp 2005; Renninger und Hidi 2016; Schiefele 2009). Krapp (2002) definiert Interesse in der sogenannten person-object theory of interest (POI) als eine „Person-Gegenstands-Beziehung“. Interesse resultiert folglich aus der Auseinandersetzung eines Individuums mit einem Gegenstand in seiner Umwelt. Dieser Gegenstand kann einem tatsächlichen Gegenstand entsprechen, aber auch einem Thema oder einer abstrakten Idee (Krapp und Prenzel 2011). Die POI ist in erster Linie geeignet, eine ontogenetische Entwicklung von Interesse zu erklären, welche per se relativ stabil ist. Weniger adäquat ist sie jedoch, um spontan auftretendes Interesse in einer Situation, bspw. während einer Lerngelegenheit im Schulkontext, zu erklären (Krapp 2002). Hierfür ist eine Betrachtung von Interesse notwendig, die genauso wie die POI zwischen einem stabilen (individuelles Interesse) und einem situationsabhängigen Anteil (situationales Interesse) differenziert (Krapp 2002), aber einen stärkeren Fokus auf das situationale Interesse und assoziierte Prozesse legt (z. B. Hidi 2001).

Individuelles Interesse entspricht prinzipiell dem Interesse, wie es in der POI formuliert wurde: Es umfasst die Prädisposition eines Individuums, sich wiederholt mit einem bestimmten Gegenstand auseinanderzusetzen, und ist damit zeitlich sehr stabil (Schiefele 2009). Bezogen auf die Naturwissenschaften wurde z. B. das Interesse der Lernenden an der Domäne Chemie bzw. am Chemieunterricht untersucht (Daniels 2008), aber auch an spezifischen Fachthemen (Hoffmann et al. 1998; Elster 2007). Weiterhin haben Dierks et al. (2014) mit dem RIASEC+N-Modell (in Anlehnung an das RIASEC-Modell zur Erfassung von Berufsinteressen nach Holland (1997)) eine Möglichkeit zur Ausdifferenzierung individueller Interessen vorgeschlagen und empirisch untersucht (Blankenburg et al. 2016; Höft et al. 2019). Dieser Ansatz ist tätigkeitsbezogen, d.h. es wird das individuelle Interesse von Lernenden an typi- 
schen naturwissenschaftlichen Tätigkeiten und Arbeitsweisen untersucht. Der Name des Modells bildet ein Akronym für die verschiedenen Dimensionen, z. B. Realistic (bspw. Experimente nach einer vorgegebenen Anleitung durchführen) oder Investigative (bspw. Experimente planen, um etwas genauer zu untersuchen).

In Abgrenzung zum individuellen Interesse beschreibt das situationale Interesse einen kurzweiligen psychologischen Zustand, welcher von erhöhter Aufmerksamkeit geprägt ist (Harackiewicz et al. 2016). Der Zusammenhang zwischen diesen beiden Arten des Interesses wurde über das Four-Phase Model of Interest Development modelliert (Hidi und Renninger 2006), das eine Entwicklung des Interesses über vier konsekutive Entwicklungsstufen beschreibt: Beginnend mit der Entstehung von situationalem Interesse (triggered situational interest) über ein andauerndes situationales Interesse (maintained situational interest) entwickelt sich aus diesem schließlich ein individuelles Interesse (emerging individual interest), welches im Verlauf der Zeit stabiler wird (well-developed individual interest). Diese verschiedenen Entwicklungsstufen des Interesses gehen mit wachsendem Wissen und einer gesteigerten Wertschätzung bezüglich des Gegenstands einher (Hidi und Renninger 2006). Während der Interessensentwicklung kann zu jedem Zeitpunkt das Interesse zurückgehen oder auf der jeweiligen Stufe stagnieren (Renninger und Hidi 2011). Beides kann durch entsprechende externale Unterstützung verhindert oder gemindert werden (Azevedo 2015). Ist bereits ein individuelles Interesse ausgeprägt, so wirkt sich dieses auch positiv auf die Entwicklung eines situationalen Interesses aus, wie generell von Tsai et al. (2008) und domänenspezifisch für die Naturwissenschaften von Ainley et al. (2002) gezeigt wurde.

Empirische Evidenz für dieses Modell liefern verschiedene Studien: Cheung (2017) untersuchte in einer qualitativen Studie beispielsweise Faktoren, die individuelles Interesse am naturwissenschaftlichen Unterricht fördern, und konnte u. a. das individuelle Interesse an den Naturwissenschaften generell sowie situative Einflüsse wie die konkrete Unterrichtsgestaltung als Einflussfaktoren identifizieren. Letztere fördern dabei zunächst situationales Interesse und dadurch indirekt auch die Entwicklung von individuellem Interesse. Palmer et al. (2017) untersuchten die Entwicklung des situationalen und individuellen Interesses von Grundschullehramtsstudierenden an Naturwissenschaften während eines zehnwöchigen Studienmoduls. Sie konnten zeigen, dass wiederholtes Erleben von situationalem Interesse während des Moduls mit einem relativ stabilen, höheren individuellen Interesse an Naturwissenschaften am Ende des Moduls einhergeht.

Um Lernende bei der Entwicklung eines stabilen individuellen Interesses zu unterstützen, sollte daher das situationale Interesse adressiert werden. Aufgrund der eingangs dargestellten Problematik, dass das Interesse von Lernenden am Chemieunterricht - und am naturwissenschaftlichen Unterricht generell - sehr gering ist bzw. im Verlauf der Sekundarstufe abnimmt, erscheint die Förderung von situationalem Interesse umso wichtiger.

\subsection{Trigger von situationalem Interesse}

Maßnahmen, die der Initiierung und der Förderung von Interesse dienen, werden unter dem Begriff ,Trigger“ zusammengefasst: „The development of a new interest 
is initiated when something catches the attention of a learner, a process called triggering“ (Renninger et al. 2019, S. 2). Im Bereich der situationalen Interessenförderung lassen sich bereits einige Trigger festhalten, die sich in verschiedenen Studien mit naturwissenschaftlichem Schwerpunkt als empirisch wirksam erwiesen haben ${ }^{1}$. In weiteren Unterrichtsfächern sind Trigger teilweise anders gewichtig oder weichen ab: In sprachlichen Fächer haben z. B. die Gestaltung von Texten einen anderen Stellenwert (Hidi 2001) und Hands-on Aktivitäten umfassen andere Tätigkeiten (z.B. das Erarbeiten von Standbildern im Sinne des szenischen Darstellens).

Der Forschungsstand im naturwissenschaftlichen Unterricht ist sehr divers: Es sind Trigger in verschiedenen unterrichtsrelevanten Bereichen, die in Anlehnung an Fend (1988) eingeteilt wurden, wie in der Administration, aber auch in der Unterrichtsgestaltung, der Lernmaterialgestaltung (,Collative Variables“), in der Beziehung zwischen Lehrkraft und Lernenden, Charakteristika der Lehrkraft sowie Aspekte der Selbstbestimmungstheorie untersucht worden. Dabei ist nicht immer eindeutig zuzuordnen, welche Trigger sich tatsächlich auf situationales und welche auf individuelles Interesse auswirken. Auch die Befundlage bezüglich der interessefördernden Wirkung ist nicht durchweg eindeutig. So werden bspw. Hands-on Aktivitäten einerseits als interessefördernd beschrieben (Shirazi 2017). Andererseits schränken Holstermann et al. (2010) diese Feststellung mit ihrer Studie im Biologieunterricht ein, in der sie Hands-on Aktivitäten in verschiedene Bereiche unterteilten und differenzierte Effekte, z. T. sogar negative Effekte, auf das Interesse feststellen konnten. Walpuski und Hauck (2017) bestätigten für den Chemieunterricht, dass nicht jedes Experiment per se für Lernende interessant ist. Sie identifizierten in ihrer Studie zunächst Verbesserungspotenzial von Experimenten im Chemieunterricht (bspw. eine stärkere Betonung der Fragestellung, des Planens und der Diskussion der Ergebnisse). In einem zweiten Schritt setzten sie diese in Experimentierphasen um und konnten ein größeres Interesse bei den Lernenden im Vergleich zur Kontrollgruppe feststellen.

Einschränkungen bezüglich der Wirksamkeit oder Wirkrichtung finden sich auch bspw. bei der Gestaltung von persönlich relevanten Unterrichtsinhalten (Renninger und Bachrach 2015). Im Zuge dieser Studie stellten Renninger und Bachrach (2015) außerdem fest, dass der Interessentrigger „Neuheit“ (novelty) zu stark vereinfacht wird, da sich dieser auf verschiedene Aspekte wie die Neuheit der Lernumgebung, der Methode oder auch des Lerninhalts beziehen kann. Zudem können verschiedene Trigger gleichzeitig auftreten, bspw. eine Kombination aus einer Hands-on Aktivität und Neuheit (der Aktivität und/oder des Inhalts). Demnach sollte ein deutlich detaillierterer Blick auf den Unterricht geworfen werden, um festzustellen, an welcher Stelle Potenziale zur Interessenförderung liegen. Bei fast allen Studien handelt es sich entweder um Interventionen, Reviews oder um post-hoc Untersuchungen von bereits stattgefundenem Unterricht. ${ }^{2}$ Erstere untersuchen per se nicht den Unterricht, der von Lehrkräften konzipiert wird, sondern analysieren die Wirksamkeit der für einen speziellen Zweck erstellten Interventionsmaterialien. Letztere hingegen können aufgrund der temporären Instabilität des situationalen Interesses nur

\footnotetext{
1 S. Tab. 1 im digitalen Anhang.

2 Eine Übersicht befindet sich im digitalen Anhang (s. Tab. 1).
} 
begrenzt analysieren, wann und wodurch dieses bei den Lernenden hervorgerufen wurde. Damit ergibt sich ein Bedarf an einer zeitlich feinkörnigeren Untersuchung des Entstehens und Aufrechterhaltens des situationalen Interesses von Lernenden im naturwissenschaftlichen Unterricht. Wenige empirische Studien haben sich diesem bereits gewidmet: Patall et al. (2016) bspw. haben die Entstehung sowie die Stabilität von situationalem Interesse über den Verlauf einer Unterrichtseinheit (aber nicht innerhalb der Unterrichtsstunden) untersucht, die Unterrichtsqualität als Beschreibung der ablaufenden unterrichtlichen Prozesse wurde dabei nicht berücksichtigt.

\subsection{Unterrichtsqualität}

Unterricht und seine Wirkung werden maßgeblich von der Lehrkraft beeinflusst, da sie diesen gestaltet und durchführt. Im Rahmen des Angebots-Nutzungs-Modells macht sie den Lernenden das Angebot (den Unterricht) (Helmke 2007). Die Nutzung dieses Angebots erfolgt in Form der Lernaktivitäten der Lernenden; der Lernzuwachs wird als Wirkung oder Ertrag klassifiziert. Im Hinblick auf das Interesse der Lernenden veranschaulicht dieses Modell, dass die Unterrichtsqualität durch die Lehrkraft und deren professionelle Kompetenz beeinflusst wird. Mögliche Kriterien zur Erfassung der Unterrichtsqualität wurden in verschiedenen Katalogen dargelegt und in unterschiedlichen Studien empirisch untersucht. Hier sind u. a. die Arbeiten von Clausen et al. (2003) oder auch die Ergebnisse des COACTIV-Projekts (Kunter und Voss 2013) zu nennen. Aktuelle Betrachtungen zeigen, dass die häufig vorgeschlagene dreidimensionale Struktur der Unterrichtsqualität (Potenzial zur kognitiven Aktivierung, Klassenführung und konstruktive Unterstützung, z. B. Kunter und Voss 2013) die einzelnen Facetten von Unterrichtsqualität sowohl im generischen als auch im fachspezifischen Bereich nicht ausreichend abbilden kann (Praetorius und Charalambous 2018).

Ein fächerübergreifendes Instrumentarium zur Beobachtung von Unterrichtsqualität bietet auch das Projekt EMU (Evidenzbasierte Methoden der Unterrichtsdiagnostik) von Helmke et al. (2010), das sich an Hatties Meta-Analyse lernförderlicher Unterrichtsmerkmale (2009) orientiert und die Bereiche Klassenführung, lernförderliches Klima und Motivierung, Klarheit und Strukturiertheit, Aktivierung und Förderung sowie eine generelle Bilanz der Unterrichtsstunde erfasst (Helmke et al. 2010; s. Tab. 1). Dieses Instrument kann im Zuge der Qualitätssicherung sowohl zur Eigen- als auch Fremdreflexion von Unterricht durch Lehrkräfte genutzt werden.

In Hatties Meta-Analyse (2009) wurden diese Kriterien in Bezug auf die Leistung der Lernenden untersucht. Sowohl die Lernzielklarheit $(d=0,75)$ als auch ein für die Lernenden fordernder Unterricht $(d=0,64)$, wie er von Helmke et al. (2010) im Bereich Aktivierung und Förderung beschrieben wird, haben einen großen Effekt auf die Leistung. Weiterhin spielt auch elaboriertes Feedback $(d=0,72)$ eine große Rolle, während die Klassenführung einen mittleren Effekt hat $(d=0,52)$ (Hattie 2009).

Generell besteht ein großer Zusammenhang zwischen fachübergreifenden und fachspezifischen Merkmalen der Unterrichtsqualität (Praetorius et al. 2020): Die universale Ebene (z.B. der Klassenführung) und die der Subdimension (z. B. Verhaltensmanagement) ist zunächst generisch zu verstehen. Auf Ebene des Indikators bzw. des Items hingegen zeigen sich bspw. für die Naturwissenschaften fachspe- 
Tab. 1 Unterrichtsqualitätsmerkmale nach Helmke et al. (2010)

\begin{tabular}{ll}
\hline Bereich & Inhalt \\
\hline Klassenführung & Zeitmanagement \\
& $\begin{array}{l}\text { Ungestörte Arbeitsatmosphäre } \\
\text { Allgegenwärtigkeit der Lehrkraft }\end{array}$ \\
Lernförderliches & Umgang mit Fehlern \\
Klima und & Feedback \\
Motivierung & Motivierende Aufgabengestaltung \\
Klarheit und & Lernzielklarheit \\
Strukturiert- & Zusammenfassung der Lerninhalts \\
heit & $\begin{array}{l}\text { Inhaltlich verständliche Erklärungen durch die } \\
\text { Lehrkraft }\end{array}$ \\
Aktivierung & Beteiligung der Lernenden am Unterrichtsgespräch \\
und & Fordernde Aufgaben \\
Förderung & Binnendifferenzierung \\
\hline
\end{tabular}

zifische Besonderheiten (z.B. Allgegenwärtigkeit, Prävention und Intervention), da hier fachdidaktisches Wissen zur Beurteilung genutzt werden muss (Heinitz und Nehring 2020). Folglich lässt sich ein generisches Instrumentarium (z.B. das des EMU-Projekts) durch die konkrete Ausformulierung der Indikatoren für bestimmte Unterrichtsfächer anpassen. Gemäß der Ausführungen im Rahmen der PISA-Studie soll naturwissenschaftlicher Unterricht strukturiert, störungsarm, kognitiv aktivierend und diszipliniert sein. Daneben soll er eine angemessene Unterstützung durch die Lehrkraft bieten, wobei diese im Gleichgewicht mit einer inhaltlichen Öffnung stehen sollte (Schiepe-Tiska et al. 2016). Diese Merkmale der Unterrichtsqualität sind bisher vor allem darauf untersucht worden, ob sie eine positive Auswirkung auf die Leistung der Lernenden haben (Hattie 2009; Stronge et al. 2007). Aufgrund des nachgewiesenen Zusammenhangs zwischen Interesse und Leistung (z. B. Schiefele et al. 1992) kann jedoch davon ausgegangen werden, dass diese Merkmale auch eine positive Wirkung auf das Interesse der Lernenden haben.

Neben Qualitätsmerkmalen ist die Gestaltung von Unterricht ein zentrales Merkmal, die u. a. durch die Wahl der Sozialform, Thematik und Methodik beschrieben werden kann. Diese sind teilweise auch als potenzielle Interessentrigger empirisch untersucht worden (z.B. Gruppenarbeit (Matthews 2004) oder Projektarbeit (Hulleman und Harackiewicz 2009)). Neben diesen methodisch-inhaltlichen Bereichen zählt auch die Phasierung von Unterricht zur Unterrichtsgestaltung (Kiper et al. 2011). Die Idee des Phasierens wurde erstmals von Roth (1957) formuliert. Seine Annahme war, dass Lernen durch ein stufenförmiges Problemlösen erfolgt. Dieses Schema wurde von Petersen et al. (2011) vereinfacht, sodass es auf jeden Unterricht übertragbar ist. Die vereinfachte Phasierung lautet: Einstieg/Motivation, Problematisierung, Erarbeitung, Sicherung und Auswertung sowie Transfer. Dabei muss nicht jede Unterrichtsstunde strikt nach diesem Schema ablaufen. Vielmehr wird Unterricht aus verschiedenen dieser Bausteine in weitgehend variabler Reihenfolge zusammengesetzt. Eine Besonderheit im Chemieunterricht ist die Durchführung von 
Experimenten. Daher lässt sich die Erarbeitung in eine theoretische und eine praktische (experimentelle) Erarbeitungsphase unterteilen. ${ }^{3}$

Die empirische Befundlage zur Wirkung von Unterrichtsphasierung ist dünn. Generell ist festzuhalten, dass die Phasierung von Unterricht größtenteils auf Erfahrungen und/oder schulpädagogischen und fachdidaktischen Theorien beruht (Petersen et al. 2011). Allerdings ist jede Phase durch bestimmte Charakteristika bezüglich der Unterrichtsgestaltung gekennzeichnet. In Einstiegsphasen werden die Lernenden häufig mit einem neuen Phänomen konfrontiert, in den praktischen Erarbeitungsphasen finden beispielsweise Experimente statt und in Sicherungs- bzw. theoretischen Erarbeitungsphasen wird häufig mehr geschrieben als in den zuerst genannten Phasen. In Verbindung mit den bereits vorgestellten Interessentriggern lässt sich daher vermuten, dass Einstiegsphasen, in denen den Lernenden neue Phänomene oder Fragestellungen vorgestellt werden, eine positive Wirkung auf das situationale Interesse der Lernenden haben, da sie den Trigger „Neuheit“ beinhalten (z. B. Palmer 2009). Diese positive Wirkung konnte von Ruppert (2010) bereits für den Biologieunterricht gezeigt werden, sodass eine Übertragung auf den Chemieunterricht wahrscheinlich ist. Weiterhin gelten theorielastige Phasen (z. B. theoretische Erarbeitungs- oder Sicherungsphasen) als hinderlich für das situationale Interesse von Lernenden (Logan und Skamp 2013). Viele Studien im Bereich der Unterrichtsphasierung fokussieren zudem eher die Seite der Lehrkräfte (z. B. Planungskompetenz, Vergleiche zwischen unterschiedlichen Lehrkräften etc.) und nicht die Lernenden (Zierer und Wernke 2017). Neben der klassischen Unterrichtsphasierung nach Roth gibt es auch weitere Unterrichtskonzeptionen wie die Basismodelle (z.B. Nutzen von Lernstrategien oder problembasiertes Lernen) nach Oser und Baeriswyl (2001). Wackermann et al. (2008) untersuchten die Wirksamkeit der Umsetzung dieses Modells im Rahmen einer Physiklehrkräftefortbildung und arbeiteten heraus, dass eine klare Umsetzung der in diesem Modell formulierten Phasen zu verbesserter Unterrichtswahrnehmung, Leistung und Emotionen bei den Lernenden führt. Je strikter die Lehrkräfte das Basismodell in ihren Unterricht integrierten, desto stärker verbesserten sich die Zuwächse bei den Lernenden. Allerdings ist dieses Modell nur ganzheitlich untersucht worden und nicht in Bezug auf einzelne Phasen.

\section{Fragestellungen}

Ziel der vorliegenden Studie ist die Analyse der Zusammenhänge von Unterrichtsphasierung sowie von Unterrichtsqualitätsmerkmalen mit dem situationalen Interesse von Lernenden im Chemieunterricht. Zur Analyse sollen die Qualitätsmerkmale von Unterricht, die von Helmke et al. (2010) im Zuge des EMU-Projekts operationalisiert worden sind (s. Abschn. 4.2), sowie eine allgemeine Protokollierung des Unterrichts genutzt werden. Insgesamt soll die übergreifende Frage, inwiefern bestimmte Merkmale unterrichtlicher Qualität in Relation mit dem situationalen Interesse der Lernenden stehen und ob sich grundsätzliche Unterschiede in der Ausprägung des

3 Die Phasen sind im digitalen Anhang (Tab. 3) ausführlicher beschrieben. 
situationalen Interesses in verschiedenen Unterrichtsphasen zeigen, untersucht werden.

FF1 Welche Unterrichtsqualitätsmerkmale sind unter Kontrolle der Zeit ${ }^{4}$ mit dem situationalen Interesse von Lernenden im Chemieunterricht assoziiert?

Anhand der bereits untersuchten Interessentrigger lassen sich einige Hypothesen zur Beantwortung dieser Forschungsfrage formulieren: Ein hohes Klassenmanagement geht vermutlich mit einem niedrigem situationalen Interesse einher, da die Lenkung des Unterrichts sehr stark ist (Stronge et al. 2007). Dieses Ergebnis wurde auch im Mathematikunterricht gefunden (Grünkorn et al. 2020). Es hat sich zudem gezeigt, dass Unterrichtsformen mit einer geringeren Lenkung durch die Lehrkraft einen positiven Effekt auf das situationale Interesse haben (Harackiewicz et al. 2016). In weiteren Studien wurde im Kontrast dazu gezeigt, dass das Klassenmanagement auch positiv mit dem Interesse der Lernenden verknüpft sein kann (z.B. Kunter et al. 2007, 2013). In beiden Studien wird angeführt, dass effektives Klassenmanagement einen positiven Einfluss auf das Interesse der Lernenden hat. In bestimmten Fällen kann aber eine hohe Ausprägung von Klassenmanagement ineffizient sein (z. B. wenn die gesamte Lernzeit für den Unterricht verwendet wird, obwohl die Lernenden konstant von einem anderen Ereignis abgelenkt werden).

Weiterhin wurde gezeigt, dass Lernende auf einer motivational-affektiven Ebene von einer hohen Unterrichtsqualität im Bereich der konstruktiven Unterstützung profitieren (Kunter et al. 2013). Folglich ist davon auszugehen, dass Aspekte, die das Lernen vereinfachen und damit in die Kategorie der konstruktiven Unterstützung fallen, wie bspw. individuelle Förderung und Verständnisorientierung, vermutlich ebenfalls im positiven Zusammenhang mit dem situationalen Interesse stehen.

FF2 Wie unterscheidet sich unter Kontrolle der Zeit die Ausprägung des situationalen Interesses von Lernenden in verschiedenen Unterrichtsphasen?

Es wurde die Phasierung nach Petersen et al. (2011) untersucht. Wenngleich eine Phasierung nicht in allen Unterrichtskonzeptionen (bspw. projektorientiertem Unterricht) die gleiche Rolle spielen dürfte, scheinen spezifische Phasen zumindest im „typischen“ naturwissenschaftlichen Unterricht, wie er in Videostudien (Björkman und Tiemann 2013; Stiller 2016) oder in Einschätzungen von Schülerinnen und Schülern (Schiepe-Tiska et al. 2016) charakterisiert wird, die vorherrschende Organisationsform zu sein. Eine dominante Phase im Chemieunterricht ist die praktische Erarbeitungsphase, in der Experimente von Lernenden aktiv oder von Lehrkräften als Demonstration durchgeführt werden können. Für diese Phasen wird ein positiver Zusammenhang erwartet. Weiterhin wird für theoretische Erarbeitungsphasen eine negative Beziehung zum situationalen Interesse erwartet und für Einstiegsphasen eine positive (s. Abschn. 4.2). Aufgrund des aktuellen Forschungsstandes können für den möglichen Zusammenhang mit den Sicherungsphasen keine Hypothese aufgestellt werden.

\footnotetext{
4 Da die Erhebung über mehrere Unterrichtsstunden an verschiedenen Schultagen erfolgte, wurde die Zeit im Sinne der Sequenz der Unterrichtshospitationen als Kontrollvariable eingefügt.
} 
FF3 Welcher Zusammenhang besteht zwischen dem individuellen Interesse der Lernenden an typischen naturwissenschaftlichen Tätigkeiten (unter Berücksichtigung der Effekte der Unterrichtsmerkmale und -phasen auf Klassenebene sowie der Zeit) und dem situationalen Interesse der Schülerinnen und Schüler im Chemieunterricht?

Ergänzend zu den Unterrichtsphasen und -merkmalen soll mit dem individuellen Interesse ein zentrales Schülermerkmal berücksichtigt und dessen Zusammenhang zum situationalen Interesse untersucht werden. Grundsätzlich wird in dem Vier-Phasen-Modell formuliert, dass situational interessierte Lernende individuelles Interesse entwickeln können (Hidi und Renninger 2006). Der umgekehrte Pfad ist theoretisch nicht vorgesehen. In verschiedenen Studien konnte aber bereits gezeigt werden, dass Lernende mit höherem individuellem Interesse auch ein höheres situationales Interesse zeigen (Ainley et al. 2002). Deswegen wird auch hier erwartet, dass individuelles Interesse im positiven Zusammenhang mit dem situationalen Interesse steht.

\section{Methodisches Vorgehen}

\subsection{Studiendesign}

Die Studie wurde an fünf Gymnasien Schleswig-Holsteins durchgeführt. Insgesamt haben 240 Lernende in neun Klassen teilgenommen. Die Schulen wurden teilweise aufgrund der Teilnahme an einem Vorgängerprojekt (,DoLiS“, Development of Learning in Science) akquiriert. Zur Vergrößerung der Stichprobe haben darüber hinaus noch zwei weitere Schulen teilgenommen. Die Stichprobe stellt demnach eine Gelegenheitsstichprobe dar. Alle Lernenden besuchten während der Durchführung die achte Jahrgangsstufe. Da die Studie parallel in allen Klassen durchgeführt wurde, wurde der gleiche Fachinhalt (Einführung in ,Salze“) unterrichtet. Diese Jahrgangsstufe wurde ausgewählt, da der Chemieunterricht hier beginnt. Für die Erhebung wurde ein Thema ausgewählt, welches bereits über den klassischen Anfangsunterricht hinausgeht.

Die Erhebungsleiterin hospitierte in jeder Klasse im Chemieunterricht, wobei die Anzahl der Hospitationen zwischen drei und sechs Schulstunden variierte ${ }^{5}$. Bei 13 der 22 Hospitationen war eine zweite Person anwesend. Beide wurden zuvor durch das Codieren und Protokollieren von Unterrichtsvideos geschult. Während der Hospitationen wurde die Ausprägung verschiedener Unterrichtsqualitätsmerkmale erhoben, der Unterricht protokolliert und die Arbeitsmaterialien erfasst. Außerdem wurde zu verschiedenen Zeitpunkten das situationale Interesse der Lernenden erhoben (s. Abschn. 6.2).

\footnotetext{
5 Die variierende Stundenanzahl ist auf organisatorische Gründe zurückzuführen, da insgesamt zu drei verschiedenen Zeitpunkten hospitiert werden sollte. In einigen Klassen fand der Unterricht in einem 60 minTakt (drei Schulstunden Hospitation) statt, während andere im Doppelstundenmodus 90 min je Woche (sechs Schulstunden Hospitation) unterrichtet wurden.
} 
Tab. 2 Zusammenfassung der Stichprobenzusammensetzung

\begin{tabular}{|c|c|c|c|c|c|c|}
\hline \multirow[t]{2}{*}{ Schule } & \multirow[t]{2}{*}{ Klasse } & \multicolumn{2}{|l|}{ Lernende } & \multirow[t]{2}{*}{ Hospitationen } & \multicolumn{2}{|l|}{ Ratings } \\
\hline & & Gesamt & Ind. Interesse & & Gesamt & Ind. Interesse \\
\hline \multirow[t]{2}{*}{$\mathrm{A}$} & A & 30 & 18 & 5 & 272 & 164 \\
\hline & B & 23 & 17 & 4 & 143 & 112 \\
\hline B & A & 25 & 21 & 6 & 143 & 129 \\
\hline \multirow[t]{3}{*}{$\mathrm{C}$} & A & 29 & 22 & 4 & 245 & 202 \\
\hline & B & 25 & 22 & 4 & 79 & 77 \\
\hline & $\mathrm{C}$ & 25 & 22 & 4 & 135 & 120 \\
\hline \multirow[t]{2}{*}{$\mathbf{J}$} & A & 25 & n.e. & 3 & 227 & n.e. \\
\hline & B & 24 & n.e. & 3 & 158 & n.e. \\
\hline $\mathrm{K}$ & A & 34 & n.e. & 4 & 223 & n.e. \\
\hline Gesamt & & 240 & 122 & & 1625 & 804 \\
\hline
\end{tabular}

Hospitationen Anzahl der hospitierten Schulstunden, Ratings Gesamtanzahl der abgegebenen Interessenratings über alle Hospitationstermine, ind. Interesse Anzahl der Lernenden, bei denen das individuelle Interesse erhoben wurde, n.e. Nicht erhoben

Um das individuelle Interesse der Lernenden zu erfassen, wurde eine Teilstichprobe gewählt, die sowohl an dieser Studie als auch an dem DoLiS-Projekt teilgenommen hat (s. Tab. 2), in dem längsschnittlich kognitive und motivational-affektive Variablen in der Sekundarstufe erfasst wurden (Höft et al. 2019). Der letzte Messzeitpunkt des Vorgängerprojekts lag ca. zwei Monate vor der Durchführung dieser Studie. Aufgrund der zeitlichen Nähe zwischen den Erhebungszeitpunkten und der angenommenen relativen Stabilität des Konstruktes werden die vorhandenen Daten zum individuellen Interesse in das Modell integriert.

\subsection{Erhebungsinstrumente}

Zur Erfassung des situationalen Interesses wurden die Lernenden nach jeder Unterrichtsphase (z. B. nach einem Einstieg) von der Lehrkraft aufgefordert, über ein Tablet auf einer vierstufigen Likert-Skala ( $1=$ uninteressant bis $4=$ sehr interessant) anzugeben, wie interessant sie die vorangegangene Unterrichtsphase fanden (adaptiert nach Palmer 2009). Vor der Unterrichtsstunde erfolgte eine Absprache mit der Lehrkraft bezüglich der Abgrenzung zwischen den Unterrichtsphasen, welche während der Unterrichtsstunde von der Lehrkraft angekündigt wurden. Es wurde eine inhaltliche Einordnung der Unterrichtsphasen in Phasen wie Einstiegs-, Sicherungsund theoretische oder praktische Erarbeitungsphasen unternommen. ${ }^{6}$

Das situationale Interesse wurde anhand eines Items erfragt, um die zeitliche Instabilität des situationalen Interesses zu berücksichtigen und möglichst wenig in den Unterricht einzugreifen. Wenngleich die Verwendung von Einzelitems keine Prüfung der Messgenauigkeit erlaubt, konnte Palmer (2009) durch zusätzliche Interviews und anschließende Datentriangulation Belege für die Validität dieser Methode vorlegen. Insgesamt wurden $N=2104$ Messungen von situationalem Interesse

\footnotetext{
${ }^{6}$ Das Codiermanual zur Abgrenzung der verschiedenen Unterrichtsphasen befindet sich im digitalen Anhang.
} 
$(M=2,82, S D=0,85)$ vorgenommen (inkl. fehlender Werte, s. Abschn. 6.3). Das entspricht ca. 17 Messungen je Lernenden.

Ferner wurden für jede Unterrichtsphase Unterrichtsqualitätsmerkmale mithilfe des Beobachtungsbogens aus dem EMU-Projekt erfasst (adaptiert nach Helmke et al. 2010) ${ }^{7}$. Durch die Anpassungen wurde der Beobachtungsbogen für den Chemieunterricht spezifiziert. Die Items wurden auf einer 4-stufigen Likert-Skala geratet. Die Abstufungen der Items wurden vor der Anwendung diskursiv validiert und mithilfe von Unterrichtsvideos verfeinert und erprobt. ${ }^{8}$ Für die Übereinstimmung mit dem zweiten Rater wurde eine Interraterreliabilität (Cohens $\kappa$ ) berechnet, welche mit $\kappa=0,88$ in einem sehr guten Bereich liegt (Wirtz und Caspar 2002).

Da die Reliabilitäten (Cronbachs $\alpha$ ) einzelner Skalen ${ }^{9}$ nicht zufriedenstellend waren und die ursprünglichen Skalen nach Helmke et al. (2010) auch in faktorenanalytischen Verfahren nicht bestätigt werden konnten, wurde eine explorative Faktorenanalyse mit non-orthogonaler Rotation (Oblimin) anhand der 23 LikertItems durchgeführt. Die mündliche Beteiligung, welche im ursprünglichen Fragebogen durch Zählen der Wortmeldungen enthalten ist, wurde nicht berücksichtigt. Der Bartlett-Test auf Sphärizität zeigte nach der Löschung von zwei Items, dass die Korrelationen zwischen den Items annehmbar sind $\left(\chi^{2}(210)=994,06, p<0,001\right)$. Der Kaiser-Meyer-Olkin-Wert liegt mit $\mathrm{KMO}=0,67[0,34 ; 0,90]$ im annehmbaren Bereich, wobei einige Einzelitems sehr niedrige Werte aufweisen (Field et al. 2013). Der Verlauf der Eigenwerte und des Scree-Plots weisen bei Anwendung des KaiserKriteriums auf eine sechs-faktorielle Struktur hin. Auf zwei Faktoren lädt allerdings jeweils nur ein Item, weswegen diese Faktoren nicht weiter berücksichtigt wurden. Ein Item zeigt Querladungen auf mehreren Faktoren, ein weiteres Item wurde aus inhaltlichen Gründen nicht weiter berücksichtigt. Die verbleibende vierfaktorielle Lösung wurde unter Ausschluss der o.g. Items wiederholt durchgeführt, wobei die Faktorzuordnung repliziert werden konnte ${ }^{10}$. Die Varianzaufklärung liegt bei $67 \%$. Die Kommunalitäten zeigen ebenfalls, dass eine vier-faktorielle Struktur angemessen ist. Die Faktoren und der Range der Einzelladungen sind in Tab. 3 zusammengefasst. Im digitalen Anhang befindet sich der Beobachtungsbogen mit den verwendeten 17 Items (Tab. 3). Diese sind den entsprechenden Skalen (Klassenmanagement etc.) zugeordnet. Einige Items sind nicht für alle Phasen gleichermaßen relevant und wurden dementsprechend ausgelassen, wenn diese für die jeweilige Phase nicht bestimmt werden konnten.

Die Erfassung des individuellen Interesses wurde während eines Vorgängerprojekts (s. oben) für eine Teilstichprobe erhoben. Hierbei wurde ein tätigkeitsbezogener Ansatz gewählt, welcher auf dem RIASEC+N-Modell beruht ${ }^{11}$ (Blankenburg et al. 2016; Dierks et al. 2014). Auf Basis dieses Modells wurden die Lernenden zu ihrem

\footnotetext{
7 Die genauen Anpassungen der Items sind im digitalen Anhang ausgeführt.

8 Das Codiermanual für die Abstufungen der verwendeten Items befindet sich im digitalen Anhang.

9 Zunächst wurde die Reliabilität der von Helmke et al. (2010) festgelegten Skalen getestet. Für die Skalen „Lernförderliches Klima und Motivierung“ sowie „Aktivierung und Förderung“ waren die Reliabilitäten nicht zufriedenstellend.

10 Die Scree-Plots befinden sich im digitalen Anhang (Abb. 1 und 2).

11 Die genutzten Items finden sich im digitalen Anhang (Tab. 4).
} 
Tab. 3 Zusammenfassung der deskriptiven Statistik und der Ergebnisse der explorativen Faktorenanalyse. (Die Items wurden adaptiert nach Helmke et al. 2010)

\begin{tabular}{lllllll}
\hline Skala & M $^{\mathrm{a}}$ & SD & Ladung & Reliabilität & $\begin{array}{l}\text { Anzahl } \\
\text { der Items }\end{array}$ & Inhalt \\
\hline $\begin{array}{l}\text { Klassen- } \\
\text { management }\end{array}$ & 2,88 & 0,53 & $0,61-0,85$ & 0,78 & 5 & $\begin{array}{l}\text { Organisation } \\
\text { Zeitmanagement } \\
\text { Allgegenwärtigkeit } \\
\text { Förderung }\end{array}$ \\
& 2,61 & 0,67 & $0,45-0,88$ & 0,80 & 6 & $\begin{array}{l}\text { Wahl eines angemessenen } \\
\text { Schwierigkeitsgrads } \\
\text { Schülerzentrierung }\end{array}$ \\
& & & & & & $\begin{array}{l}\text { Umgang mit Fehlern } \\
\text { Verständnis- } \\
\text { orientierung }\end{array}$ \\
& 3,19 & 0,49 & $0,32-0,63$ & 0,69 & 3 & $\begin{array}{l}\text { Feedback } \\
\text { Fordernde Aufgaben } \\
\text { Beteiligung der Lernenden am } \\
\text { Unterrichtsgespräch } \\
\text { rierung }\end{array}$ \\
& 3,05 & 0,76 & $0,56-0,86$ & 0,73 & 3 & $\begin{array}{l}\text { Sicherung des Lernstoffs } \\
\text { Verwendung verschiedener } \\
\text { Darstellungsformen }\end{array}$ \\
\hline
\end{tabular}

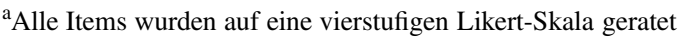

Interesse an der Durchführung typischer naturwissenschaftlicher Unterrichtstätigkeiten befragt. Die Gesamtskala auf der Basis von 29 Items $(M=2,84, S D=0,46)$, welche auf einer 4-stufigen Likert-Skala geratet wurden, weist eine Reliabilität von $\alpha=0,92$ auf.

\subsection{Datenauswertung}

Um adäquat mit fehlenden Werten auf der Ebene des situationalen und individuellen Interesses umzugehen, wurden multiple Imputationen durchgeführt, da dieses Vorgehen herkömmlichen Methoden wie listen- oder paarweisem Fallausschluss überlegen ist (Newman 2014). Fehlende Werte entstanden hauptsächlich durch vollständiges Fehlen einzelner Lernender in einer Unterrichtsstunde (z. B. durch Krankheit) oder durch das zufällige Verpassen einzelner Abstimmungen für das situationale Interesse, sodass kein Zusammenhang mit anderen Konstrukten vermutet wird. Daher wurden die multiplen Imputationen unter der Annahme MAR (missing at random) in der Statistiksoftware R mit den Paketen „,mitml“ und „,mice“ durchgeführt (Grund et al. 2019; R Core Team 2018; van Buuren und Groothuis-Oudshoorn 2011). Die Imputationen wurden nach dem Prinzip des joint modelling durchgeführt. Das dazugehörige Imputationsmodell entspricht für MLA 1 der detailliertesten Version der Mehrebenenanalyse, welche im Ergebnisteil dargestellt wird (Tab. 6, Mod1.3). Für die Teilstichprobe liegen weitere Daten aus dem DoLiS-Projekt vor. Da nur ein Teil der Stichprobe an diesen Erhebungen teilgenommen hat, konnte keine globale Imputation für alle Forschungsfragen durchgeführt werden. Daher wurde für diese Imputation über die detaillierteste Mehrebenenanalyse (Tab. 8, Mod2.4) hinaus das naturwissenschaftliche Selbstkonzept, die Chemienote, das Geschlecht und die generelle Lernfreude als Hilfsvariablen im Imputationsmodell berücksichtigt. Durch die 
Verwendung eines überlappenden Sets an Hilfsvariablen (Skalen der Unterrichtsqualität usw.) folgen beide Imputationen einer inklusiven Strategie, sodass die Annahme „MAR“ nicht verletzt wird (Collins et al. 2001). Die enge Passung zwischen Forschungsfrage und Imputationsmodell wiederum entspricht der Empfehlung aktueller Studien, auch wenn dann mehrfache Imputationen notwendig werden (vgl. Enders et al. 2020; Lüdtke et al. 2020). Der prozentuale Anteil der fehlenden Werte liegt für das situationale Interesse in der Mehrebenenanalyse mit der vollständigen Stichprobe bei 22,8\%. Für die Teilstichprobe liegt der Anteil bei 23,6\% und der Anteil der fehlenden Werte für das individuelle Interesse bei 6,3\%. Die fehlenden Werte wurden auf Skalenebene imputiert. Die FMI-Werte (fraction of missing information) geben den Anteil der Informationen für eine Variable an, die aufgrund der Imputation verloren geht (Enders 2010). Sie liegen für diese Imputation im Bereich von 0,005 bis 0,301 . Die potential scale reduction entspricht 1 . Beide Werte liegen damit im gutem bis sehr gutem Bereich (Grund et al. 2016). ${ }^{12}$

Um die Koeffizienten der Mehrebenenanalyse miteinander vergleichen zu können, wurden die Skalen der Unterrichtsqualität, des situationalen und des individuellen Interesse standardisiert. Nach der Imputation wurden die Werte zunächst anhand des Gruppenmittelwertes mit der Klasse als Gruppierungsvariable zentriert und anschlieBend der Mittelwert für das individuelle Interesse gebildet. Durch dieses Vorgehen konnte die Clusterung der Lernenden in verschiedenen Klassen berücksichtigt werden, obwohl die Stichprobe auf Klassenebene für eine explizite Implementierung in die Mehrebenenanalysen zu klein ist (Enders und Tofighi 2007; Snijders und Bosker 2012). Da die Erhebung über mehrere Unterrichtsstunden an verschiedenen Schultagen erfolgte, wurde die Zeit als Kontrollvariable eingefügt.

Um die hierarchisch geschachtelte Struktur (Abb. 1) zu berücksichtigen, wurden Mehrebenenanalysen mit dem Paket „Ime4“ in R durchgeführt (Bates et al. 2015). Mehrebenenanalysen sind lineare Regressionen, die verschiedene Level von Daten (Abb. 1) berücksichtigen und somit Fehlinterpretationen vermeiden können. Aufgrund der verhältnismäßig geringen Stichprobengröße wurden Random-InterceptModelle gerechnet (Snijders und Bosker 2012). Die ICC liegt für die erste Mehrebenenanalyse (Forschungsfragen 1 und 2) bei 0,123 und für die zweite Mehrebenenanalyse (Forschungsfrage 3) bei 0,139, d.h. 12,3\% bzw. 13,9\% der Varianz liegen zwischen den Personen, was für eine Berücksichtigung der Mehrebenen-Struktur spricht.

Nach der multiplen Imputation wurden zudem die Korrelationen zwischen dem situationalen Interesse der Lernenden, den Unterrichtsmerkmalen und den Unterrichtsphasen berechnet. Bei der Untersuchung der Unterrichtsphasen wurden nur diejenigen Phasen untersucht, die mehr als acht Mal beobachtet werden konnten. Das betrifft die Phasen Einstieg, theoretische Erarbeitungsphase, praktische Erarbeitungsphase und Sicherung; die drei Phasen Problematisierung, Transfer und Wie-

\footnotetext{
12 In den burn-in-Phasen wurden 50.000 Iterationen durchgeführt. Anschließend wurden 100 imputierte Datensätze mit 5000 Iterationen erstellt. Um die Ergebnisse über die imputierten Datensätze hinweg zu bündeln, wurden die im R-Paket mitml (Grund et al. 2016) implementierte Rubin-Regeln (1987) verwendet.
} 
Abb. 1 Leveleinteilung für die Mehrebenenanalyse; da die Stichprobe auf Level 3 zu klein ist, wird die Klassenebene nicht explizit modelliert, sondern durch Zentrierung der Variablen auf den Klassenmittelwert (group-mean centering) berücksichtigt

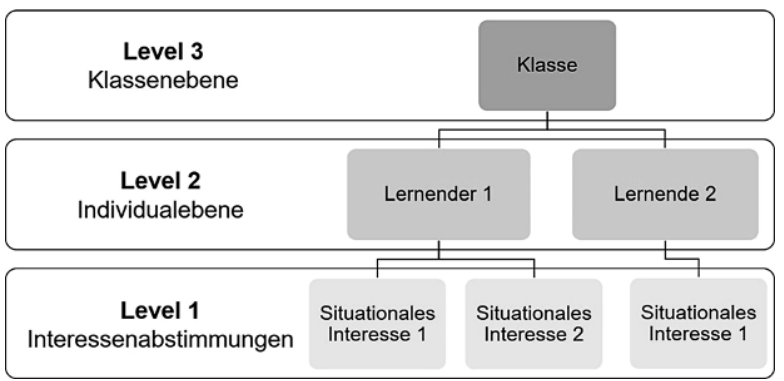

Tab. 4 Pearson-Korrelationen ( $r$ ) der erhobenen Variablen; in Klammern ist der Standardfehler $(S E)$ angegeben

\begin{tabular}{|c|c|c|c|c|c|}
\hline & SI & KM & $\mathrm{F}$ & $\mathrm{V}$ & $\mathrm{S}$ \\
\hline $\begin{array}{l}\text { Situationales Interes- } \\
\text { se (SI) }\end{array}$ & 1 & - & - & - & - \\
\hline $\begin{array}{l}\text { Klassenmanagement } \\
(\mathrm{KM})\end{array}$ & $\begin{array}{l}-0,16^{* * * *} \\
(0,02)\end{array}$ & 1 & - & - & - \\
\hline Förderung (F) & $\begin{array}{l}0,04 \\
(0,02)\end{array}$ & $\begin{array}{l}0,21 * * * \\
(0,02)\end{array}$ & 1 & - & - \\
\hline Verständnis (V) & $\begin{array}{l}-0,08^{* * * *} \\
(0,02)\end{array}$ & $\begin{array}{l}-0,01 \\
(0,02)\end{array}$ & $\begin{array}{l}0,03 \\
(0,02)\end{array}$ & 1 & - \\
\hline Strukturierung (S) & $\begin{array}{l}0,07 * * \\
(0,02)\end{array}$ & $\begin{array}{l}-0,37 * * * \\
(0,02)\end{array}$ & $\begin{array}{l}0,00 \\
(0,02)\end{array}$ & $\begin{array}{l}0,41 * * * \\
(0,02)\end{array}$ & 1 \\
\hline Einstieg & $\begin{array}{l}-0,05^{*} \\
(0,02)\end{array}$ & $\begin{array}{l}0,05^{*} \\
(0,02)\end{array}$ & $\begin{array}{l}-0,07 \\
(0,02)^{* *}\end{array}$ & $\begin{array}{l}-0,24 * * * \\
(0,02)\end{array}$ & $\begin{array}{l}-0,45^{* * * *} \\
(0,02)\end{array}$ \\
\hline $\begin{array}{l}\text { Praktische Erarbei- } \\
\text { tung }\end{array}$ & $\begin{array}{l}0,19 * * * \\
(0,02)\end{array}$ & $\begin{array}{l}-0,53 * * * \\
(0,02)\end{array}$ & $\begin{array}{l}-0,16^{* * * *} \\
(0,02)\end{array}$ & $\begin{array}{l}0,11 * * * \\
(0,02)\end{array}$ & $\begin{array}{l}0,42 * * * \\
(0,02)\end{array}$ \\
\hline $\begin{array}{l}\text { Theoretische Erar- } \\
\text { beitung }\end{array}$ & $\begin{array}{l}-0,08 * * * \\
(0,02)\end{array}$ & $\begin{array}{l}0,10 * * * \\
(0,02)\end{array}$ & $\begin{array}{l}0,16 * * * \\
(0,02)\end{array}$ & $\begin{array}{l}0,08 * * * \\
(0,02)\end{array}$ & $\begin{array}{l}0,22 * * * \\
(0,02)\end{array}$ \\
\hline Sicherung & $\begin{array}{l}0,04 \\
(0,02)\end{array}$ & $\begin{array}{l}0,31 * * * \\
(0,02)\end{array}$ & $\begin{array}{l}0,05^{*} \\
(0,02)\end{array}$ & $\begin{array}{l}0,08 * * * \\
(0,02)\end{array}$ & $\begin{array}{l}-0,02 \\
(0,02)\end{array}$ \\
\hline
\end{tabular}

Signifikanzniveau der Koeffizienten $* * * p<0,001, * * p<0,01, * p<0,05$

derholung wurden entsprechend von den Analysen ausgeschlossen. In Tab. 4 sind die Korrelationen zusammengefasst.

\section{Ergebnisse}

\subsection{Zusammenhang zwischen situationalem Interesse und Unterrichtsmerkmalen}

Die Ergebnisse der Mehrebenenanalyse sind in Tab. 6 zusammengefasst: Mod1.0 und Mod1.1 dienen der Kontrolle: Mod1.0 entspricht dem Nullmodell ohne Prädiktoren, während Mod1.1 die Kontrollvariable Zeit als Prädiktor enthält. Die Zeit wurde als Sequenz der Unterrichtshospitationen (d.h. erster Hospitationstermin = 1, zweiter Hospitationstermin $=2$ usw.) codiert. Die Zeit weist dabei einen negativen 
Tab. 5 Ergebnisse des Wald-Tests zum Vergleich des Modell-Fits für Modell 1. Genaue Spezifikationen der Modelle können der Tab. 6 entnommen werden

\begin{tabular}{lllllll}
\hline Modellvergleich & & F-Wert & df1 & df2 & $p$ & RIV \\
\hline Mod1.1 & Mod1.0 & 0,88 & 1 & 6005,41 & 0,35 & 0,14 \\
Mod1.2 & Mod1.0 & 19,52 & 5 & $13.898,41$ & 0,00 & 0,23 \\
Mod1.2 & Mod1.1 & 23,74 & 4 & 9592,72 & 0,00 & 0,25 \\
Mod1.3 & Mod1.2 & 15,97 & 4 & $16.616,30$ & 0,00 & 0,18 \\
\hline
\end{tabular}

Zusammenhang zum situationalen Interesse auf. Mit Blick auf Forschungsfrage 1 wurde untersucht, in welchem Zusammenhang die Unterrichtsmerkmale Klassenmanagement, Verständnisorientierung, Strukturierung und Förderung zum situationalen Interesse der Lernenden stehen (Mod1.2). Entsprechend der Erwartung zeigt sich beim Klassenmanagement ein negativer Zusammenhang zum situationalen Interesse $\left(\gamma_{10}=-0,29, p<0,001\right)$. Ein weiterer negativer Zusammenhang zum situationalen Interesse liegt, entgegen der Hypothese, bei der Verständnisorientierung vor $\left(\gamma_{40}=-0,21, p<0,001\right)$. Positive Zusammenhänge wurden für die Skalen Förderung $\left(\gamma_{30}=0,20, p<0,05\right)$ und Strukturierung $\left(\gamma_{20}=0,12, p<0,05\right)$ gefunden.

Die Varianzaufklärung $\mathrm{R}^{2}(\mathrm{SB})$ ist speziell für die Anwendung in Mehrebenenanalysen entwickelt und gibt die Gesamtaufklärung des Modells unter Berücksichtigung der verschiedenen Level an (Snijders und Bosker 2012). Die Varianzaufklärung liegt in diesem Modell bei $\mathrm{R}^{2}(\mathrm{SB})=0,060$, also ca. 6,0\%. Bei Betrachtung der Varianzaufklärung auf Levelebene ergibt sich auf Level 1 eine Aufklärung von ca. 8,0\%. Auf Level 2 ist eine negative Varianzaufklärung zu beobachten, welche bei Mehre-

Tab. 6 Ergebniszusammenfassung der Mehrebenenanalyse (Mod1.0-Mod1.3) auf Basis der Gesamtstichprobe $(N=240)$

\begin{tabular}{|c|c|c|c|c|c|c|c|c|c|}
\hline \multirow[b]{2}{*}{ Feste Effekte } & \multirow[b]{2}{*}{ Parameter } & \multicolumn{2}{|c|}{ Mod1.0 } & \multicolumn{2}{|c|}{ Mod1.1 } & \multicolumn{2}{|l|}{ Mod1.2 } & \multicolumn{2}{|l|}{ Mod1.3 } \\
\hline & & $\beta$ & SE & $\beta$ & SE & $\beta$ & SE & $\beta$ & SE \\
\hline Intercept & $\gamma 00$ & 0,00 & 0,03 & 0,02 & 0,04 & 0,08 & 0,04 & 0,00 & 0,05 \\
\hline Zeit & $\gamma_{01}$ & - & - & $-0,04$ & 0,04 & $-0,12 * *$ & 0,04 & $-0,13 * * *$ & 0,04 \\
\hline Klassenmanagement & $\gamma_{10}$ & - & - & - & - & $-0,29 * * *$ & 0,05 & $-0,16^{* *}$ & 0,05 \\
\hline Strukturierung & $\gamma_{20}$ & - & - & - & - & $0,12 * *$ & 0,04 & $0,05 * *$ & 0,05 \\
\hline Förderung & $\gamma_{30}$ & - & - & - & - & $0,20 * *$ & 0,07 & $0,23 * * *$ & 0,07 \\
\hline Verständnisorientierung & $\gamma_{40}$ & - & - & - & - & $-0,21 * * *$ & 0,04 & $-0,22 * * *$ & 0,04 \\
\hline $\begin{array}{l}\text { Erarbeitungsphase } \\
\text { (praktisch) }\end{array}$ & $\gamma_{50}$ & - & - & - & - & - & - & $0,44 * * *$ & 0,07 \\
\hline $\begin{array}{l}\text { Erarbeitungsphase } \\
\text { (theoretisch) }\end{array}$ & $\gamma 60$ & - & - & - & - & - & - & $-0,19 *$ & 0,09 \\
\hline Sicherungsphase & $\gamma 70$ & - & - & - & - & - & - & 0,06 & 0,07 \\
\hline Einstiegsphase & $\gamma_{80}$ & - & - & - & - & - & - & $-0,12$ & 0,08 \\
\hline \multicolumn{10}{|l|}{ Varianzaufklärung } \\
\hline$R_{\text {Level 1 }}^{2}$ & & 0 & & 0,017 & & 0,080 & & 0,120 & \\
\hline$R_{\text {Level } 2}^{2}$ & & 0 & & 0,017 & & $-0,082$ & & $-0,246$ & \\
\hline$R_{1}^{2}(\mathrm{SB})$ & & 0 & & 0,017 & & 0,060 & & 0,076 & \\
\hline
\end{tabular}

Signifikanzniveau der Koeffizienten $* * * p<0,001, * * p<0,01, * p<0,05$ 
benenanalysen darauf zurückzuführen ist, dass keiner der Prädiktoren auf Level 2 wirkt (Snijders und Bosker 2012). Der Wald-Test zeigt, dass Mod1.2 signifikant besser auf die Daten passt als das Mod1.0 und Mod1.1 (s. Tab. 5).

\subsection{Zusammenhänge von inhaltlichen Unterrichtsphasen und dem situationalen Interesse}

Bei Ergänzung der berücksichtigten Unterrichtsphasen (s. Abschn. 6.3) als einzelne Dummy-Variablen im Modell ändern sich teilweise die Zusammenhänge der Unterrichtsmerkmale (s. Tab. 6, Mod1.3). Besonders stark ändert sich der Zusammenhang zwischen Klassenmanagement und situationalem Interesse, welcher von $\gamma_{10}=-0,29, p<0,001$ auf $\gamma_{10}=-0,16, p<0,001$ sinkt. Daher variiert der Zusammenhang zwischen Klassenmanagement und situationalem Interesse der Lernenden in Abhängigkeit von der Unterrichtsphase. Die eingangs dargestellten Korrelationen zwischen Unterrichtsmerkmalen und -phasen (s. Tab. 4) zeigen, dass das Klassenmanagement in Sicherungsphasen höher ausgeprägt ist $(r=0,31, p<0,001)$, während es in den praktischen Erarbeitungsphasen tendenziell am schwächsten ausgeprägt ist $(r=-0,53, p<0,001)$.

Mit Blick auf Zusammenhänge der einzelnen Unterrichtsphasen zum situationalen Interesse zeigt sich erwartungskonform, dass für theoretische Erarbeitungsphasen ein negativer Zusammenhang $\left(\gamma_{60}=-0,19, p<0,05\right)$ vorliegt, während für praktische Erarbeitungsphasen ein großer positiver Zusammenhang $\left(\gamma_{50}=0,44, p<0,001\right)$ besteht. Die übrigen Unterrichtsphasen (Sicherungen und Einstiege) weisen keine signifikanten Zusammenhänge zum situationalen Interesse auf. Die Varianzaufklärung vergrößert sich durch das Berücksichtigen der Unterrichtsphasen insgesamt $\left(\mathrm{R}^{2}(\mathrm{SB})=0,076\right)$ und auf Level $1\left(R_{\text {Level } 1}^{2}=0,120\right)$. Insgesamt werden auf Level 1 somit $12,0 \%$ der Varianz durch die Unterrichtsqualitätsmerkmale und -phasen aufgeklärt. Auf Level 2 vergrößert sich das negative R ${ }^{2}$. Für Mod1.3 zeigt der WaldTest bessere Fit-Werte als für Mod1.2 (s. Tab. 5).

\subsection{Zusammenhänge zwischen individuellem und situationalem Interesse}

Die zweite Mehrebenenanalyse gleicht der ersten im Kern. Es wurde nur eine Teilstichprobe berücksichtigt. Die Prädiktoren wurden in der gleichen Reihenfolge eingefügt wie bei der ersten Mehrebenenanalyse zur Beantwortung der Forschungsfragen 1 und 2 (s. Mod1.0 bis Mod1.3). Es zeigen sich tendenziell ähnliche Zusammenhänge. Generell sind die Zusammenhänge niedriger bzw. bleiben teilweise nicht signifikant (Tab. 8). Letzteres trifft auf die Zusammenhänge zwischen dem Klassenmanagement $\left(\gamma_{10}=-0,09, p=0,271\right)$, der Förderung $\left(\gamma_{30}=-0,08, p=0,464\right) \mathrm{bzw}$. der praktischen Erarbeitungsphase $\left(\gamma_{50}=0,18, p=0,081\right)$ und dem situationalen Interesse der Lernenden zu. Für die Verständnisorientierung bleibt der Zusammenhang hingegen gleich $\left(\gamma_{40}=-0,40, p>0,001\right)$. Die Varianzaufklärung ist ähnlich zu der in Mod1.3. Bei Einfügen des individuellen Interesses als Level 2-Variable zeigt sich ein großer Zusammenhang zum situationalen Interesse $\left(\gamma_{02}=0,27 * * *, p<0,001\right)$. Durch die Aufnahme dieser Variable nimmt die Varianzaufklärung deutlich zu: Insgesamt werden $10,9 \%$ der Varianz aufgeklärt $\left(\mathrm{R}^{2}(\mathrm{SB})=0,109\right)$ und auf Level 2 
Tab. 7 Ergebnisse des Wald-Tests zum Vergleich des Modell-Fits für die zweite Mehrebenenanalyse. Genaue Spezifikationen der Modelle können der Tab. 8 entnommen werden

\begin{tabular}{lllllll}
\hline Modellvergleich & & F-Wert & df1 & df2 & $p(>\mathrm{F})$ & RIV \\
\hline Mod2.1 & Mod2.0 & 5,74 & 1 & 5886,91 & 0,02 & 0,14 \\
Mod2.2 & Mod2.1 & 13,37 & 4 & 6690,25 & 0,00 & 0,32 \\
Mod2.3 & Mod2.2 & 5,82 & 2 & $34.751,14$ & 0,00 & 0,08 \\
Mod2.4 & Mod2.3 & 18,77 & 1 & 1888,15 & 0,00 & 0,28 \\
\hline
\end{tabular}

$21,4 \%\left(R_{\text {Level } 2}^{2}=0,214\right)$. Die Varianzaufklärung auf Level 1 wird wie erwartet etwas kleiner $\left(R_{\text {Level } 1}^{2}=0,093\right)$. Der Wald-Test zeigt bessere Fit-Werte für Mod2.4 (s. Tab. 7).

\section{Diskussion}

Die erste Zielsetzung dieser Studie war es, Aspekte der Unterrichtsqualität zu identifizieren, die in einem engen Zusammenhang zum situationalen Interesse der Lernenden stehen. Während für die Merkmale Strukturierung und Förderung eine positive Beziehung zum situationalen Interesse der Lernenden festgestellt wurde, wurde für Klassenmanagement und Verständnisorientierung eine negative Beziehung diagnostiziert. Die zweite Zielsetzung adressierte die additive Analyse der Zusammenhänge zwischen Unterrichtsphasen und situationalem Interesse der Lernenden. Dabei zeigten sich für praktische Erarbeitungsphasen positive und für theoretische Erarbeitungsphasen negative Zusammenhänge zum situationalen Interesse der Lernenden. Für die übrigen analysierten Unterrichtsphasen (Einstieg und Sicherung) konnten im Rahmen der hier beobachteten Unterrichtsstunden keine bedeutsamen Relationen festgestellt werden.

Die dritte Zielsetzung war es, zu untersuchen, inwiefern motivational-affektive Variablen, welche bereits dispositional vorliegen, auf Individualebene einen Einfluss auf die Ausprägung des situationalen Interesses im Unterricht haben. Es wurde erwartungskonform ein substanzieller positiver Zusammenhang festgestellt. Als Kontrollvariable wurde die Zeit als Sequenz der Hospitationstermine berücksichtigt. Die negative Beziehung deutet auf ein grundsätzlich nachlassendes situationales Interesse über den Verlauf der Termine hin. Zurückzuführen ist dieses vermutlich auf einen nachlassenden Reiz des zu Beginn noch neuen Themas „Salze“ oder auf eine Gewöhnung an die Erhebungsmethode des situationalen Interesses über die Tablets. Beide Faktoren zielen im Endeffekt auf den möglichen Interessentrigger „Neuheit“, der aber nur für die ersten Begegnungen mit Thema oder Methode gelten kann (Lin et al. 2013; Palmer 2009).

\subsection{Unterrichtsmerkmale und -phasen}

Die untersuchten Zusammenhänge des situationalen Interesses mit Merkmalen der Unterrichtsqualität entsprechen für Klassenmanagement, Förderung und Strukturierung den eingangs formulierten Hypothesen. Konträr zu den Erwartungen ist der 


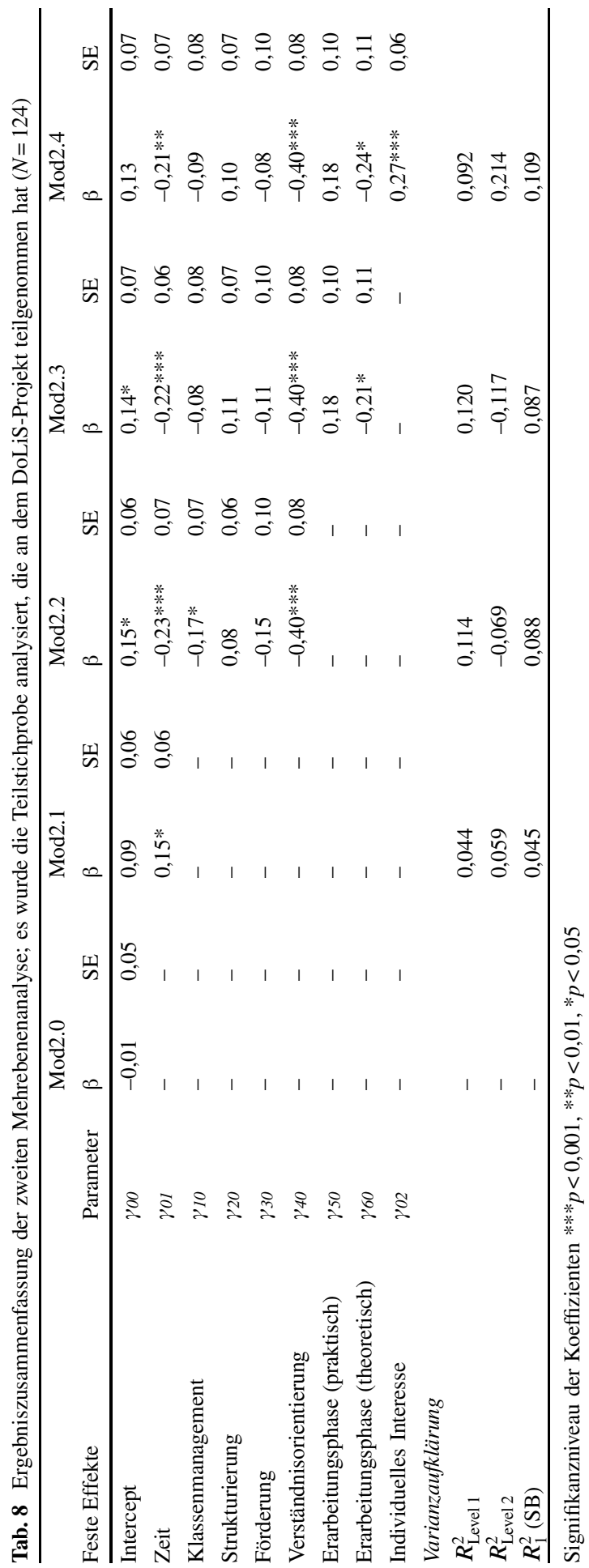


Befund, dass Verständnisorientierung eine negative Beziehung zu dem situationalen Interesse hat. Unter Verständnisorientierung wurden Aspekte wie eine angemessene Schwierigkeit und das Feedback-Geben an die Lernenden subsumiert. Auch wenn Forschungsbefunde zum Feedback zeigen, dass dieses einen positiven Einfluss auf Interesse hat (Harks et al. 2013), muss einschränkend festgehalten werden, dass die Qualität und Art des Feedbacks über sein Potenzial zur Interessenförderung entscheiden (Hattie und Timperley 2007). Beides wurde in der vorliegenden Studie nicht erfasst. Daher ist es möglich, dass die relevanten Aspekte zum Feedback hoch geratet wurden, weil sie im Unterricht häufig vorkamen, aber die Qualität dieser Aspekte nicht hoch genug war, um das situationale Interesse der Lernenden zu fördern.

Die beobachteten Zusammenhänge zwischen dem situationalen Interesse der Lernenden und den Unterrichtsphasen fallen überwiegend erwartungskonform aus und entsprechen den Ergebnissen anderer Studien. Holstermann et al. (2010) haben bspw. die Wirkung von praktischen Aufgaben im Biologieunterricht untersucht und festgestellt, dass insbesondere die Qualität der Aufgaben einen positiven Effekt auf das Interesse von Lernenden hat. In der vorliegenden Studie scheinen die praktischen Aufgaben von einer ausreichenden Qualität gewesen zu sein, dass sie interessefördernd wirkten. Eine weitere Differenzierung von Experimenten in eine rezeptartige Durchführung und eine Durchführung nach eigenständiger Planung konnte nicht vorgenommen werden, da erstere deutlich überwogen. Auch wenn Höft et al. (2019) eine hohe Korrelation zwischen den Interessen der Lernenden an diesen beiden Arten der Experimente beschreiben, stellten Walpuski und Hauck (2017) fest, dass Experimente mit einem hohen Anteil an eigener Aktivität (z. B. beim Planen und Auswerten) als interessanter wahrgenommen werden als Experimente mit rezeptartiger Anleitung.

Theoretische Erarbeitungsphasen gehen in der vorliegenden Studie eher mit einem niedrigen situationalen Interesse einher. Hier scheint aus unterrichtspraktischer Perspektive noch besonderes Potenzial zu bestehen, um den Unterricht für Lernende motivierender zu gestalten. Um diese Potenziale zu konkretisieren, müssen diese Phasen jedoch in weiterführenden Studien detaillierter inhaltlich analysiert werden (vgl. Ochsen et al. 2020).

Für Einstiegsphasen wurde kein systematischer Zusammenhang zum situationalen Interesse der Lernenden gefunden, obwohl eine positive Beziehung zum situationalen Interesse denkbar gewesen wäre, wenn in diesen Phasen den Lernenden z. B. unbekannte Phänomene oder Probleme präsentiert werden (Palmer 2009; Ruppert 2010). Das dadurch induzierte situationale Interesse kann im Verlauf des weiteren Unterrichts wieder abfallen, da die anfangs unbekannten Phänomene sukzessive mit neuem Wissen aufgeklärt werden. Diese Beobachtung haben Rotgans und Schmidt (2017) in der „knowledge-deprivation-hypothesis“ formuliert. Die Voraussetzung zur Entstehung von situationalem Interesse ist demnach ein (mit dem aktuellen Wissensstand) unlösbares Problem bzw. eine unerklärbares Phänomen. Eine mögliche Ursache für das vorliegende Ergebnis könnte also sein, dass die Einstiegsproblematiken entweder nicht motivierend genug waren oder den Lernenden nicht als Problem bewusst wurden. Merritt et al. (2017) betonen in ihrem Review ebenfalls, dass die Strukturierung und Präsentation des Problems ein wesentlicher Gelingensfaktor von problembasiertem Unterricht darstellen. Einstiegsphasen müssen aber nicht zwangs- 
läufig ein unerklärbares Phänomen präsentieren. Sie können auch das Wiederholen der letzten Unterrichtsinhalte forcieren. Diese reine Wiederholung adressiert keinen bekannten Interessentrigger und kann demzufolge als weniger interessefördernd eingestuft werden. Für die beobachteten Einstiegsphasen könnte gelten, dass sie eher diesem Muster gefolgt sind.

Bei kombinierter Berücksichtigung von Unterrichtsmerkmalen und -phasen in den Modellen halbiert sich der Zusammenhang zwischen Klassenmanagement und situationalem Interesse fast. Dies deutet auf eine Wechselwirkung mit den Unterrichtsphasen hin. Deskriptiv sind relativ hohe Korrelationen (Tab. 5) zwischen Klassenmanagement und Sicherungsphasen bzw. praktischen Erarbeitungsphasen zu finden, wobei letztere Korrelation negativ ist. Die Ausprägung des Klassenmanagements scheint somit abhängig von der Unterrichtsphase zu sein und insbesondere in praktischen Erarbeitungsphasen niedriger auszufallen.

Die Varianz, welche durch das Modell insgesamt aufgeklärt wird, ist eher niedrig. Sie weist darauf hin, dass weitere Faktoren das situationale Interesse beeinflussen, die über die untersuchten Merkmale des Unterrichts hinausgehen. Dazu könnten die Sozialform (z.B. Gruppenarbeit als interessefördernde Sozialform, Jack und Lin 2017), bestimmte Methoden wie bspw. Quizze (Bicen und Kocakoyun 2018) oder schülerzentrierte Ansätze wie Think-Pair-Share (Renninger et al. 2019) sowie weitere zeitliche und organisatorische Rahmenbedingungen (u.a. Klausuren oder Probefeueralarm) gehören.

\subsection{Individuelles Interesse}

Der hier festgestellte positive Zusammenhang zwischen dem individuellen und dem situationalen Interesse wird von weiteren Studien bestätigt (z. B. Ainley et al. 2002). Es gibt allerdings Anzeichen dafür, dass eine differenzierte Analyse der Beziehung zwischen situationalem und individuellem Interesse angebracht sein könnte: Rotgans und Schmidt (2018) haben festgestellt, dass der Effekt nur zu Beginn einer Unterrichtseinheit besonders stark ist und im Verlauf nachlässt. Im Rahmen der vorgenommenen Modellierung wäre es denkbar, den Einfluss der Zeit als Interaktion mit dem individuellen Interesse zu berücksichtigen, um herauszufinden, inwiefern der Fortgang einer Unterrichtseinheit den Einfluss des individuellen Interesses auf das situationale Interesse beeinflusst. Dazu wäre jedoch eine größere Stichprobe notwendig gewesen, weswegen diese These hier nicht geprüft werden konnte. Dennoch konnte mit der vorliegenden Studie gezeigt werden, dass das individuelle Interesse auch nach Berücksichtigung unterrichtlicher Faktoren inkrementell einen großen Anteil der Varianz im situationalen Interesse der Lernenden aufklärt und somit nicht vernachlässigt werden sollte.

Durch die Aufnahme des individuellen Interesses in das Modell wird die Varianzaufklärung deutlich erhöht. Dies ist ein weiteres Indiz dafür, dass individuelles Interesse in einem starken Zusammenhang mit dem situationalen Interesse steht. Dennoch ist dieser Zusammenhang kein Hinweis darauf, dass die Unterrichtsgestaltung eine untergeordnete Rolle bei gleichzeitiger Betrachtung des individuellen Interesses einnimmt. Da dieses aus dem situationalen Interesse entsteht, betont es vielmehr die Wichtigkeit, durch eine externale Unterstützung situationales Interes- 
se hervorzurufen (Hidi und Renninger 2006). Somit wird die Rolle der Lehrkraft bei der Unterrichtsgestaltung betont, denn es besteht die unmittelbare Möglichkeit, durch den Unterricht nachhaltig das Interesse am Fach zu fördern.

\section{Limitationen und Fazit}

Vor der Betrachtung der Implikationen dieser Studie soll zunächst auf einige Limitationen hingewiesen werden. Zum einen betrifft dies die begrenzte Stichprobenziehung und -größe. Die Stichprobe beschränkt sich auf Gymnasien in SchleswigHolstein und stellt in erster Linie eine Gelegenheitsstichprobe dar. Durch die begrenzte Stichprobengröße war es nicht sinnvoll möglich, Interaktionseffekte zwischen verschiedenen Prädiktoren, bspw. zwischen individuellem Interesse bzw. Unterrichtsqualitätsmerkmalen und Unterrichtsphasen, zu analysieren. Insofern wurden alle Prädiktoren im Modell in erster Linie additiv betrachtet und im Hinblick auf ihren individuellen Beitrag zur Aufklärung der Varianz des situationalen Interesses der Lernenden untersucht. Wechselwirkungen zwischen diesen Prädiktoren, die insbesondere aus theoretischer Sicht relevante Moderationsannahmen in den Blick genommen hätten, konnten entsprechend nicht untersucht werden. Dies wäre aber ein wünschenswerter Fokus für weiterführende Studien, die auf eine umfangreichere Stichprobe zurückgreifen können.

Auch auf die Stichprobengröße zurückzuführen ist die Begrenzung auf RandomIntercept-Modelle. Weiterhin wurde nur eine Variable (individuelles Interesse) auf Individualebene untersucht. Die Einbeziehung weiterer individueller Konstrukte wie dem naturwissenschaftlichen Selbstkonzept oder Leistungsmaßen, aber auch dem Geschlecht wären sinnvolle Ergänzungen, um detailliertere Einsichten in die interindividuelle Wahrnehmung von Interessentrigger zu gewinnen. Auf intraindividueller Ebene ist die Einbeziehung weiterer Prädiktoren potenziell interessant (bspw. Lernfreude, s. Abschn. 8.1), um die geringe Varianzaufklärung zu erhöhen.

Mit Blick auf die Erhebungsmethode ist festzuhalten, dass das situationale Interesse nur mithilfe eines Items erfragt wurde. Diese methodische Entscheidung wurde insbesondere mit dem Ziel getroffen, das situationale Interesse möglichst direkt im Unterrichtsgeschehen zur erfassen und den Unterrichtsablauf nur geringfügig zu stören. Dennoch wurde damit in den Unterricht eingegriffen und eine künstliche Pause geschaffen. Weiterhin konnte für dieses Messinstrument keine Messgenauigkeit bestimmt werden. In der Studie von Palmer (2009), aus der diese Erhebungsmethode adaptiert wurde, konnte die Validität der Ergebnisinterpretation jedoch mittels qualitativer Interviews bestätigt werden.

Letztlich ist auch die Unterrichtsbeobachtung (anstelle bspw. einer Videographie) kritisch zu reflektieren. Eine Videographie hätte mehr Analysepotenzial am Unterrichtsgeschehen ermöglicht, aber auch den zeitlichen und organisatorischen Aufwand insbesondere mit Blick auf die Teilnahme der Lernenden deutlich erhöht. Limitierend sind die fehlenden Werte für das situationale Interesse zu benennen, welche jedoch mithilfe multipler Imputationen angemessen berücksichtigt wurden.

Um die begonnene Mikroanalyse von Unterricht konsequent weiterzuführen, ist es notwendig, eine differenziertere Analyse der Unterrichtsqualitätsmerkmale vor- 
zunehmen, die neben der Quantität auch die Qualität der einzelnen Faktoren stärker berücksichtigt. Diese Untersuchung kann als qualitative Fortsetzung zur Auswertung dieser Studie durchgeführt werden. Im Rahmen des Chemieunterrichts gibt es bereits viele Unterrichtskonzeptionen (z. B. Oser und Baeriswyl 2001; Parchmann et al. 2006; Schiepe-Tiska et al. 2016), in denen die Phasen teilweise inhaltlich anders gestaltet sind. Da in der vorliegenden Studie allerdings keine dieser Konzeptionen stringent im Unterricht durchgeführt wurde, gelten unsere Ergebnisse nicht für die Phasierungen innerhalb dieser Konzeptionen. Weitere Untersuchungen zeigen, dass die genannten Konzeptionen generell wenig im Unterricht eingesetzt werden (Jannack et al. 2019), sodass die vorliegende Studie durchaus repräsentativ sein könnte. Eine weitere Limitation dieser Studie ist die Begrenzung auf den Chemieunterricht. Dieser kann vermutlich aufgrund seiner strukturellen Ähnlichkeit als Repräsentant naturwissenschaftlicher Unterrichtsfächer gelten. Für andere Unterrichtsfächer ist die Übertragbarkeit jedoch explizit zu prüfen.

Als Implikationen für Schulpraxis und Forschung lassen sich Hinweise aufzeigen, an welchen Stellschrauben Potenzial zur weiteren Interessenförderung besteht, wie bspw. im Bereich des Klassenmanagements oder durch eine Umgestaltung der Einstiegsphasen. Auch die theoretische Erarbeitungsphase wirkt in der vorliegenden Studie nicht interessefördernd. Eine Möglichkeit, diesem Befund zu begegnen, wäre eine stärkere aktive Einbindung der Lernenden in dieser Phase. Dies könnte durch theoretische Aufgaben wie Recherchen etc. erreicht werden (Hidi et al. 2015). Genauso scheint für das Unterrichtsmerkmal Verständnisorientierung die Möglichkeit $\mathrm{zu}$ bestehen, Unterricht zu verändern und interessanter für Lernende zu gestalten. Gleichzeitig stellt sich auch heraus, dass bereits einige Aspekte erfolgreich implementiert sind, die eine positive Auswirkung auf Interesse haben. Hierzu gehören die praktischen Erarbeitungsphasen und Unterrichtsqualitätsmerkmale wie Strukturierung. Ein weiterer Schritt kann bspw. eine qualitative Analyse der vorliegenden Daten sein, um konkrete Ansatzpunkte zu identifizieren, die situationales Interesse von Lernenden fördern können (vgl. Ochsen et al. 2020).

Zusatzmaterial online Zusätzliche Informationen sind in der Online-Version dieses Artikels (https://doi. org/10.1007/s42010-021-00133-8) enthalten.

Funding Open Access funding enabled and organized by Projekt DEAL.

Open Access Dieser Artikel wird unter der Creative Commons Namensnennung 4.0 International Lizenz veröffentlicht, welche die Nutzung, Vervielfältigung, Bearbeitung, Verbreitung und Wiedergabe in jeglichem Medium und Format erlaubt, sofern Sie den/die ursprünglichen Autor(en) und die Quelle ordnungsgemäß nennen, einen Link zur Creative Commons Lizenz beifügen und angeben, ob Änderungen vorgenommen wurden.

Die in diesem Artikel enthaltenen Bilder und sonstiges Drittmaterial unterliegen ebenfalls der genannten Creative Commons Lizenz, sofern sich aus der Abbildungslegende nichts anderes ergibt. Sofern das betreffende Material nicht unter der genannten Creative Commons Lizenz steht und die betreffende Handlung nicht nach gesetzlichen Vorschriften erlaubt ist, ist für die oben aufgeführten Weiterverwendungen des Materials die Einwilligung des jeweiligen Rechteinhabers einzuholen.

Weitere Details zur Lizenz entnehmen Sie bitte der Lizenzinformation auf http://creativecommons.org/ licenses/by/4.0/deed.de. 


\section{Literatur}

Ainley, M., Hillman, K., \& Hidi, S. (2002). Gender and interest processes in response to literary texts. Situational and individual interest. Learning and Instruction, 12(4), 411-428.

Azevedo, F. S. (2015). Sustaining interest-based participation in science. In K. A. Renninger, M. Nieswandt \& S. Hidi (Hrsg.), Interest in mathematics and science learning (S. 281-296). Washington, DC: American Educational Research Association.

Bates, D., Mächler, M., Bolker, B., \& Walker, S. (2015). Fitting linear mixed-effects models using lme4. Journal of Statistical Software. https://doi.org/10.18637/jss.v067.i01.

Bicen, H., \& Kocakoyun, S. (2018). Perceptions of students for gamification approach. Kahoot as a case study. International Journal of Emerging Technologies in Learning (ijet), 13(02), 72-93.

Björkman, J., \& Tiemann, R. (2013). Teaching Patterns of Scientific Inquiry: A Video Study of Chemistry Lessons in Germany and Sweden. Science Education Review Letters. Advance online publication. https://doi.org/10.18452/8207.

Blankenburg, J.S., Höffler, T. N., \& Parchmann, I. (2016). Fostering today what is needed tomorrow. Investigating students' interest in science. Science education, 100(2), 364-391. https://doi.org/10.1002/ sce. 21204.

van Buuren, S., \& Groothuis-Oudshoorn, K. (2011). mice. Multivariate imputation by chained equations in R. Journal of Statistical Software. https://doi.org/10.18637/jss.v045.i03.

Cheung, D. (2017). The key factors affecting students' individual interest in school science lessons. International Journal of Science Education, 30(4), 1-23. https://doi.org/10.1080/09500693.2017. 1362711.

Clausen, M., Reusser, K., \& Klieme, E. (2003). Unterrichtsqualität auf der Basis hoch-inferenter Unterrichtsbeurteilungen. Ein Vergleich zwischen Deutschland und der deutschsprachigen Schweiz. Unterrichtswissenschaft, 31(2), 122-141.

Collins, L.M., Schafer, J.L., \& Kam, C.-M. (2001). A comparison of inclusive and restrictive strategies in modern missing data procedures. Psychological methods, 6(4), 330-351. https://doi.org/10.1037// 1082-989X.6.4.330.

Daniels, Z. (2008). Entwicklung schulischer Interessen im Jugendalter. Pädagogische Psychologie und Entwicklungspsychologie, Bd. 69. Münster: Waxmann. Diss

Dierks, P. O., Höffler, T. N., \& Parchmann, I. (2014). Profiling interest of students in science. Learning in school and beyond. Research in Science \& Technological Education, 32(2), 97-114. https://doi.org/ 10.1080/02635143.2014.895712.

Eitemüller, C., \& Walpuski, M. (2018). Wahl- und Abwahlprofile im Fach Chemie. Ergebnisse einer Clusteranalyse zur Charakterisierung von Lernenden am Ende der Sekundarstufe I. Zeitschrift für Didaktik der Naturwissenschaften, 24(1), 251-263. https://doi.org/10.1007/s40573-018-0087-6.

Elster, D. (2007). In welchen Kontexten sind naturwissenschaftliche Inhalte für Jugendliche interessant? Ergebnisse der ROSE-Erhebung in Österreich und Deutschland. PLUS LUCIS, 3, 2-8.

Enders, C. K. (2010). Applied missing data analysis. Guilford.

Enders, C.K., \& Tofighi, D. (2007). Centering predictor variables in cross-sectional multilevel models. A new look at an old issue. Psychological methods, 12(2), 121-138. https://doi.org/10.1037/1082989X.12.2.121.

Enders, C. K., Du, H., \& Keller, B. T. (2020). A model-based imputation procedure for multilevel regression models with random coefficients, interaction effects, and nonlinear terms. Psychological methods, 25(1), 88-112. https://doi.org/10.1037/met0000228.

Fend, H. (1988). Schulqualität. Die Wederentdeckung der Schule als pädagogische Gestaltungsebene. Neue Sammlung, 28(4), 537-547.

Field, A., Miles, J., \& Field, Z. (2013). Discovering statistics using R (Reprint). Los Angeles: SAGE.

Grund, S., Lüdtke, O., \& Robitzsch, A. (2016). Multiple imputation of multilevel missing data. An introduction to the R package pan. SAGE Open. https://doi.org/10.1177/2158244016668220.

Grund, S., Robitzsch, A., \& Lüdtke, O. (2019). mitml [Computer software]. https://cran.r-project.org/ package=mitml. Zugegriffen: 24. Juli 2019.

Grünkorn, J., Klieme, E., Praetorius, A.-K., \& Schreyer, P. (Hrsg.). (2020). Mathematikunterricht im internationalen Vergleich. Ergebnisse aus der TALIS-Videostudie Deutschland. Frankfurt am Main: DIPF I Leibniz-Institut für Bildungsforschung und Bildungsinformation 2020.

Harackiewicz, J. M., Smith, J.L., \& Priniski, S. J. (2016). Interest matters. The importance of promoting interest in education. Policy Insights from the Behavioral and Brain Sciences, 3(2), 220-227. https:// doi.org/10.1177/2372732216655542. 
Harks, B., Rakoczy, K., Hattie, J., Besser, M., \& Klieme, E. (2013). The effects of feedback on achievement, interest and self-evaluation. The role of feedback's perceived usefulness. Educational Psychology, 34(3), 269-290. https://doi.org/10.1080/01443410.2013.785384.

Hattie, J.A.C. (2009). Visible learning. A synthesis of over 800 meta-analyses relating to achievement (Reprinted.). London: Routledge.

Hattie, J., \& Timperley, H. (2007). The power of feedback. Review of Educational Research, 77(1), 81-112. https://doi.org/10.3102/003465430298487.

Heinitz, B., \& Nehring, A. (2020). Kriterien naturwissenschaftsdidaktischer Unterrichtsqualität - ein systematisches Review videobasierter Unterrichtsforschung. Unterrichtswissenschaft, 48(3), 319-360. https://doi.org/10.1007/s42010-020-00074-8.

Helmke, A. (2007). Unterrichtsqualität erfassen, bewerten, verbessern. Dieses Buch ist Franz-Emanuel Weinert gewidmet (Schulisches Qualitätsmanagement (6. Aufl.). Seelze: Klett.

Helmke, A., Helmke, T., Lenske, G., Pham, G., Praetorius, A.-K., Schrader, F., \& Ade-Thurow, M. (2010). Studienbrief Unterrichtsdiagnostik. Projekt Udikom der Kultusministerkonferenz. Landau: Universität Koblenz-Landau.

Hidi, S. (2001). Interest, Reading, and Learning: Theoretical and Practical Considerations. Educational Psychology Review, 13(3), 191-209. https://doi.org/10.1023/A:1016667621114

Hidi, S., \& Renninger, K. A. (2006). The four-phase model of interest development. Educational Psychologist, 41(2), 111-127. https://doi.org/10.1207/s15326985ep4102_4.

Hidi, S., Renninger, K. A., \& Nieswandt, M. (2015). Emerging issues and themes in addressing interest in learning mathematics and science. In K. A. Renninger, M. Nieswandt \& S. Hidi (Hrsg.), Interest in mathematics and science learning (S. 385-396). Washington, DC: American Educational Research Association.

Hoffmann, L., Häußler, P., \& Lehrke, M. (1998). Die IPN-Interessenstudie Physik (IPN: Vol. 158). Kiel: IPN.

Höft, L., Bernholt, S., Blankenburg, J.S., \& Winberg, M. (2019). Knowing more about things you care less about. Cross-sectional analysis of the opposing trend and interplay between conceptual understanding and interest in secondary school chemistry. Journal of Research in Science Teaching, 56(2), 184-210. https://doi.org/10.1002/tea.21475.

Holland, J. L. (1997). Making vocational choices: a theory of vocational personalities and work environments (3. Aufl.). : Psychological Assessment Resources.

Holstermann, N., Grube, D., \& Bögeholz, S. (2010). Hands-on activities and their influence on students' interest. Research in Science Education, 40(5), 743-757. https://doi.org/10.1007/s11165-009-91420 .

Hulleman, C. S., \& Harackiewicz, J. M. (2009). Promoting interest and performance in high school science classes. Science, 326(5958), 1410-1412. https://doi.org/10.1126/science.1178712.

Jack, B. M., \& Lin, H.-S. (2017). Making learning interesting and its application to the science classroom. Studies in Science Education, 23(1), 1-28. https://doi.org/10.1080/03057267.2017.1305543.

Jannack, V., Knemeyer, J.-P., \& Marmé, N. (2019). Forschungsnahe Methoden im naturwissenschaftlichen Unterricht. Lehrer/innenbefragung zeigt akuten Handlungsbedarf. heiEDUCATION Journal. Transdisziplinäre Studien zur Lehrerbildung. https://doi.org/10.17885/heiup.heied.2019.4.24024.

Kiper, H., Meyer, H., Topsch, W., \& Hinz, R. (2011). Einführung in die Schulpädagogik (6. Aufl.). Studium kompakt Unterricht, Schule. Berlin: Cornelsen.

Krapp, A. (2002). Structural and dynamic aspects of interest development. Theoretical considerations from an ontogenetic perspective. Learning and Instruction, 12(4), 11-11. https://doi.org/10.1016/S09594752 .

Krapp, A. (2005). Basic needs and the development of interest and intrinsic motivational orientations. Learning and Instruction, 15(5), 381-395.

Krapp, A., \& Prenzel, M. (2011). Research on Interest in Science. Theories, methods, and findings. International Journal of Science Education, 33(1), 27-50. https://doi.org/10.1080/09500693.2010.518645.

Kunter, M., \& Voss, T. (2013). The model of instructional quality in COACTIV. A Multicirteria analysis. In M. Kunter, J. Baumert, W. Blum, U. Klusmann, S. Krauss \& M. Neubrand (Hrsg.), Cognitive activation in the mathematics classroom and professional competence of teachers. Results from the COACTIV project. Mathematics Teachers Education, (Bd. 8, S. 97-124). New York: Springer.

Kunter, M., Baumert, J., \& Köller, O. (2007). Effective classroom management and the development of subject-related interest. Learning and Instruction, 17(5), 494-509. https://www.sciencedirect.com/ science/article/pii/S095947520700093X. 
Kunter, M., Klusmann, U., Baumert, J., Richter, D., Voss, T., \& Hachfeld, A. (2013). Professional competence of teachers. Effects on instructional quality and student development. Journal of Educational Psychology, 105(3), 805-820.

Lin, H.-S., Hong, Z.-R., \& Chen, Y.-C. (2013). Exploring the development of college students' situational interest in learning science. International Journal of Science Education, 35(13), 2152-2173. https:// doi.org/10.1080/09500693.2013.818261.

Logan, M.R., \& Skamp, K.R. (2013). The impact of teachers and their science teaching on students' 'science interest'. A four-year study. International Journal of Science Education, 35(17), 2879-2904. https://doi.org/10.1080/09500693.2012.667167.

Lüdtke, O., Robitzsch, A., \& West, S. G. (2020). Regression models involving nonlinear effects with missing data. A sequential modeling approach using Bayesian estimation. Psychological methods, 25(2), 157-181.

Lyons, T., \& Quinn, F. (2010). Choosing science. Understanding the declines in senior high school science enrolments. Armidale: National Centre of Science, Information and Communication Technology, and Mathematics Education for Rural and Regional Australia.

Maltese, A. V., \& Tai, R.H. (2011). Pipeline persistence. Examining the association of educational experiences with earned degrees in STEM among U.S. students. Science Education, 95(5), 877-907. https:// doi.org/10.1002/sce.20441.

Matthews, B. (2004). Promoting emotional literacy, equity and interest in science lessons for 11-14 year olds; the 'improving science and emotional development' project. International Journal of Science Education, 26(3), 281-308. https://doi.org/10.1080/0950069032000097406.

Merritt, J., Lee, M. Y., Rillero, P., \& Kinach, B. M. (2017). Problem-based learning in K-8 mathematics and science education. A literature review. Interdisciplinary Journal of Problem-Based Learning. https:// doi.org/10.7771/1541-5015.1674.

Newman, D. A. (2014). Missing data. Organizational Research Methods, 17(4), 372-411. https://doi.org/ $10.1177 / 1094428114548590$.

Ochsen, S., Bernholt, S., Bernholt, A., \& Parchmann, I. (2020). Eine Mikroanalyse von Chemieunterricht - Einsatz und Perzeption von Triggern für situationales Interesse. Zeitschrift für Didaktik der Naturwissenschaften, 36(1), 4. https://doi.org/10.1007/s40573-020-00122-x.

Oser, F. K., \& Baeriswyl, F. J. (2001). Choreographies of teaching: bridging instruction to learning. In V. Richardson (Hrsg.), Handbook of research on teaching 4. Aufl. Washington, DC: American Educational Research Assoc.

Palmer, D.H. (2009). Student interest generated during an inquiry skills lesson. Journal of Research in Science Teaching, 46(2), 147-165. https://doi.org/10.1002/tea.20263.

Palmer, D., Dixon, J., \& Archer, J. (2017). Using situational interest to enhance individual interest and science-related behaviours. Research in Science Education, 47(4), 731-753. https://doi.org/10.1007/ s11165-016-9526-X.

Parchmann, I., Gräsel, C., Baer, A., Nentwig, P., Demuth, R., \& Ralle, B. (2006). "Chemie im Kontext”. A symbiotic implementation of a context-based teaching and learning approach. International Journal of Science Education, 28(9), 1041-1062. https://doi.org/10.1080/09500690600702512.

Patall, E. A., Vasquez, A.C., Steingut, R.R., Trimble, S.S., \& Pituch, K. A. (2016). Daily interest, engagement, and autonomy support in the high school science classroom. Contemporary Educational Psychology, 46, 180-194. https://doi.org/10.1016/j.cedpsych.2016.06.002.

Petersen, J., Reisas, S., \& Tanski, G. (2011). Unterricht vorbereiten und planen können. Ein Lehrbuch zur Unterrichtsvorbereitung und Stundenplanung ; mit interaktiver Lern-DVD (3. Aufl.). Didaktische Welten Lehrkompetenzen, Teil 1. Augsburg: Brigg Pädagogik Verl.

Potvin, P., \& Hasni, A. (2014). Interest, motivation and attitude towards science and technology at K-12 levels. A systematic review of 12 years of educational research. Studies in Science Education, 50(1), 85-129. https://doi.org/10.1080/03057267.2014.881626.

Praetorius, A.-K., \& Charalambous, C. Y. (2018). Classroom observation frameworks for studying instructional quality. Looking back and looking forward. ZDM, 50(3), 535-553. https://doi.org/10.1007/ s11858-018-0946-0.

Praetorius, A.-K., Herrmann, C., Gerlach, E., Zülsdorf-Kersting, M., Heinitz, B., \& Nehring, A. (2020). Unterrichtsqualität in den Fachdidaktiken im deutschsprachigen Raum - zwischen Generik und Fachspezifik. Unterrichtswissenschaft, 48(3), 409-446. https://doi.org/10.1007/s42010-020-00082-8.

$\mathrm{R}$ Core Team (2018). R: A language and environment for statistical computing [Computer software]. Vienna: R Core Team. https://www.r-project.org/. 
Renninger, K.A., \& Bachrach, J.E. (2015). Studying triggers for interest and engagement using observational methods. Educational Psychologist, 50(1), 58-69. https://doi.org/10.1080/00461520.2014. 999920.

Renninger, K.A., \& Hidi, S. (2011). Revisiting the conceptualization, measurement, and generation of interest. Educational Psychologist, 46(3), 168-184. https://doi.org/10.1080/00461520.2011.587723.

Renninger, K. A., \& Hidi, S. (2016). The power of interest for motivation and engagement. New York: Routledge.

Renninger, K. A., Bachrach, J.E., \& Hidi, S. E. (2019). Triggering and maintaining interest in early phases of interest development. Learning, Culture and Social Interaction, 23, 100260.

Rotgans, J.I., \& Schmidt, H. G. (2017). The role of interest in learning:. Knowledge acquisition at the intersection of situational and individual interest. In P. A. O'Keefe \& J. M. Harackiewicz (Hrsg.), The science of interest. Cham: Springer.

Rotgans, J. I., \& Schmidt, H. G. (2018). How individual interest influences situational interest and how both are related to knowledge acquisition. A microanalytical investigation. The Journal of Educational Research, 111(5), 530-540. https://doi.org/10.1080/00220671.2017.1310710.

Roth, H. (1957). Pädagogische Psychologie des Lehrens und Lernens. Berlin, Hannover, Darmstadt: Schroedel.

Ruppert, W. (2010). Welches Interesse haben Schüler an biologischen Themen? In U. Spörhase-Eichmann \& W. Ruppert (Hrsg.), Biologie-Didaktik. Praxishandbuch für die Sekundarstufe I und II (4. Aufl. S. 107-123). Berlin: Cornelsen.

Schiefele, U. (2009). Interest and learning from text. Scientific Studies of Reading, 3(3), 257-279.

Schiefele, U., Krapp, A., \& Winterer, A. (1992). Interest as a predictor of academic achievement. A metaanalysis of research. In K. A. Renninger, S. Hidi \& A. Krapp (Hrsg.), The role of interest in learning and development (S. 183-212). Hillsdale: Lawrence Erlbaum.

Schiepe-Tiska, A., Schmidtner, S., Müller, K., Heine, J.-H., Neumann, K., \& Lüdtke, O. (2016). Naturwissenschaftlicher Unterricht in Deutschland in PISA 2015 im internationalen Vergleich. In K. Reiss, C. Sälzer, A. Schiepe-Tiska, E. Klieme \& O. Köller (Hrsg.), PISA 2015. Eine Studie zwischen Kontinuität und Innovation. Münster: Waxmann.

Shirazi, S. (2017). Student experience of school science. International Journal of Science Education, 39(14), 1891-1912. https://doi.org/10.1080/09500693.2017.1356943.

Snijders, T. A. B., \& Bosker, R. J. (2012). Multilevel analysis. An introduction to basic and advanced multilevel modeling (2. Aufl.). Los Angeles: SAGE.

Stiller, J. (2016). Scientific Inquiry im Chemieunterricht. Berlin: Humboldt-Universität zu Berlin, Mathematisch-Naturwissenschaftliche Fakultät.

Stronge, J.H., Ward, T. J., Tucker, P. D., \& Hindman, J. L. (2007). What is the relationship between teacher quality and student achievement? An exploratory study. Journal of Personnel Evaluation in Education, 20(3-4), 165-184. https://doi.org/10.1007/s11092-008-9053-z.

$\mathrm{Su}, \mathrm{R}$. (2018). The three faces of interests: An integrative review of interest research in vocational, organizational, and educational psychology. Journal of Vocational Behavior, 116(4), 103240. https://doi. org/10.1016/j.jvb.2018.10.016.

Tsai, Y.-M., Kunter, M., Lüdtke, O., Trautwein, U., \& Ryan, R. M. (2008). What makes lessons interesting? The role of situational and individual factors in three school subjects. Journal of Educational Psychology, 100(2), 460-472. https://doi.org/10.1037/0022-0663.100.2.460.

Wackermann, R., Trendel, G., \& Fischer, H.E. (2008). Überprüfung der Wirksamkeit einer lernwegeorientierten Fortbildung für Physiklehrer. In E.-M. Lankes (Hrsg.), Pädagogische Professionalität als Gegenstand empirischer Forschung (S. 61-86). Münster: Waxmann.

Walpuski, M., \& Hauck, A. (2017). Experimente und Lernerfolg. Wie können Experimentierphasen optimiert werden, um Interesse und Lernerfolg der Schülerinnen und Schüler zu erhöhen? Unterricht Chemie 2016(158), 8-13.

Wirtz, M.A., \& Caspar, F. (2002). Beurteilerübereinstimmung und Beurteilerreliabilität. Methoden zur Bestimmung und Verbesserung der Zuverlässigkeit von Einschätzungen mittels Kategoriensystemen und Ratingskalen. Göttingen: Hogrefe.

Zierer, K., \& Wernke, S. (Hrsg.). (2017). Die Unterrichtsplanung: Ein in Vergessenheit geratener Kompetenzbereich?! Status Quo und Perspektiven aus Sicht der empirischen Forschung. Bad Heilbrunn: Julius Klinkhardt. 